\title{
Multivariate High-Frequency-Based Volatility (HEAVY) Models
}

\author{
DiaA Noureldin \\ Department of Economics, University of Oxford, \\ $\& 3$ Oxford-Man Institute of Quantitative Finance, \\ Eagle House, Walton Well Road, Oxford OX2 6ED, UK \\ diaa.noureldin@economics.ox.ac.uk \\ Neil Shephard \\ Department of Economics, University of Oxford, \\ 83 Oxford-Man Institute of Quantitative Finance, \\ Eagle House, Walton Well Road, Oxford OX2 6ED, UK \\ neil.shephard@economics.ox.ac.uk \\ Kevin Sheppard \\ Department of Economics, University of Oxford, \\ 83 Oxford-Man Institute of Quantitative Finance, \\ Eagle House, Walton Well Road, Oxford OX2 6ED, UK \\ kevin.sheppard@economics.ox.ac.uk
}

February 18, 2011

\begin{abstract}
This paper introduces a new class of multivariate volatility models that utilizes high-frequency data. We discuss the models' dynamics and highlight their differences from multivariate GARCH models. We also discuss their covariance targeting specification and provide closed-form formulas for multi-step forecasts. Estimation and inference strategies are outlined. Empirical results suggest that the HEAVY model outperforms the multivariate GARCH model out-of-sample, with the gains being particularly significant at short forecast horizons. Forecast gains are obtained for both forecast variances and correlations.
\end{abstract}

Keywords: HEAVY model; GARCH; multivariate volatility; realized covariance; covariance targeting; multi-step forecasting; Wishart distribution.

JEL classification: C32; C52; C58. 


\section{Introduction}

This paper introduces a new class of multivariate volatility models capable of producing precise multi-step forecasts of the conditional covariance matrix of daily returns. Multivariate volatility models have been the focus of a voluminous literature summarized recently by Bauwens et al. (2006) and Asai et al. (2006), where the focus in the latter is on multivariate stochastic volatility.

The covariance matrix of daily asset returns is a key input in portfolio allocation, option pricing and financial risk management. An interesting question is whether the increasing availability of high-frequency financial data enables the development of more accurate forecasting models for the conditional covariance of daily returns. We address this question by studying a new class of models which utilize high-frequency data for the objective of multi-step volatility forecasting. We call this class multivariate High-frEquency-bAsed VolatilitY (HEAVY) models.

Volatility forecasts from HEAVY models have some properties that distinguish them from those of multivariate GARCH models. HEAVY models have a relatively short response time which means they are likely to perform well in periods where the level of volatility or correlation is subject to abrupt changes. HEAVY models also have short-run momentum effects so that volatility forecasts may exhibit a continuation of upward (or downward) trends in volatility before mean reverting.

The univariate HEAVY model was introduced in Shephard and Sheppard (2010) where it is shown - for a wide spectrum of asset classes - that the HEAVY model outperforms the GARCH model in- and out-of-sample. The forecast gains tend to be more pronounced at short forecast horizons, typically the first few days. In the empirical section of this paper, we show similar results in a multivariate setting. The multivariate analysis poses additional interesting questions such as whether the forecast gains are due to the variance forecasts of individual assets, their correlations or a combination of both. We develop a novel out-of-sample model evaluation strategy to address this question.

To highlight the distinction between HEAVY and GARCH models, and how HEAVY models differ from recently proposed models which also utilize high-frequency data, we start with a brief overview of the univariate HEAVY model of Shephard and Sheppard (2010). Let $\mathcal{F}_{t}^{L F}$ and $\mathcal{F}_{t}^{H F}$ respectively denote the information set generated by low-frequency (i.e. daily) and high-frequency (i.e. intra-daily) data up to time $t$, where $t=1,2, \ldots$, indexes days. Also let $r_{t}$ denote the (demeaned) daily return and $v_{t}$ denote the realized measure (e.g. realized variance) at time $t$. The univariate HEAVY model in its linear specification is the 2-equation system

$$
\begin{gathered}
\mathrm{E}\left[r_{t}^{2} \mid \mathcal{F}_{t-1}^{H F}\right]:=h_{t}=c_{h}+b_{h} h_{t-1}+a_{h} v_{t-1}, \\
\mathrm{E}\left[v_{t} \mid \mathcal{F}_{t-1}^{H F}\right]:=m_{t}=c_{m}+b_{m} m_{t-1}+a_{m} v_{t-1},
\end{gathered}
$$

while the GARCH model is

$$
\mathrm{E}\left[r_{t}^{2} \mid \mathcal{F}_{t-1}^{L F}\right]:=h_{t}^{*}=c_{g}+b_{g} h_{t-1}^{*}+a_{g} r_{t-1}^{2}
$$


The primary distinction between HEAVY and GARCH models is the conditioning information set used in modelling the conditional variance of daily returns. The first equation of the HEAVY model uses the lagged realized measure, $v_{t-1}$, to drive to dynamics of $h_{t}$, whereas the GARCH model uses the lagged squared return. The second equation of the HEAVY model is needed for multi-step forecasts of $h_{t}$.

The HEAVY model utilizes recently developed estimators of ex post volatility of daily returns that have proven to be more precise compared to squared returns. Realized variance is the first realized measure to be systematically studied and used in modelling and forecasting the volatility of daily returns. Andersen and Bollerslev (1998) show that the realized variance has a much lower noise-to-signal ratio than the daily squared return when used as proxies for the unobserved variance, while Barndorff-Nielsen and Shephard (2002) formalize the econometrics of the realized variance. In the context of multi-step forecasting, Shephard and Sheppard (2010) show that the use of the realized kernel of Barndorff-Nielsen et al. (2008) leads to notable in- and out-of-sample improvements in predicting $h_{t}$, especially at short forecast horizons.

Univariate HEAVY models are related to recently proposed models by Engle (2002), Engle and Gallo (2006), Cipollini et al. (2007), Brownlees and Gallo (2010) and Hansen et al. (2011). Engle (2002) models volatility using a multiplicative error model (MEM). ${ }^{1}$ He applies this model to squared returns and realized volatility as separate models, but they were not considered as a system for multi-step forecasting of the conditional variance of daily returns. These models are usually referred to as GARCH-X models when both $v_{t-1}$ and $r_{t-1}^{2}$ appear in the $h_{t}$ equation. Engle and Gallo (2006) model a 3-variable system comprising the squared return, the high-minus-low price range and the realized variance in an MEM setup. Cipollini et al. (2007) allow for contemporaneous correlations in a 4-variable vector MEM including the absolute daily return and three realized measures, and tackle the problem of a suitable multivariate density choice using copulas.

The papers by Brownlees and Gallo (2010) and Hansen et al. (2011) are the closest in structure to the univariate HEAVY model. The model in Brownlees and Gallo (2010) has a HEAVY-like structure with the difference being that it uses a smoothed version of the realized measure to drive $h_{t}$ by specifying the latter as an affine function of $m_{t}$. Hansen et al. (2011) treat the dynamics of the realized measure differently. While the HEAVY model postulates GARCH-type dynamics for the realized measure by modelling its conditional expectation, Hansen et al. (2011) relate the realized measure itself to $h_{t}$ and a term that captures leverage effects.

Multivariate volatility models are becoming increasingly important not only because of their direct application in portfolio allocation and asset pricing, but also due to the insights they provide into risk management practices. Using low-frequency data, Brownlees and Engle (2010) portray the importance of modelling conditional correlations for systemic risk management, where they show that a rise in a firm's stock volatility and correlation with the market magnifies its contribution to

\footnotetext{
${ }^{1}$ An MEM can be used for any non-negative valued process which can be modelled as i.i.d. innovations from a density with non-negative support scaled by a conditionally deterministic factor.
} 
systemic risk. Highly leveraged financial companies in the recent financial crisis are a case in point. The work of Hansen et al. (2010), which is independent and concurrent, utilizes realized measures in modelling a stock's conditional beta in a GARCH-like framework. Our primary empirical example focuses on the returns of Bank of America and an S\&P 500 exchange traded fund during the recent financial crisis, which relates to the applications in these papers.

There is some recent research that focuses only on modelling and forecasting the realized covariance matrix; see, for example, Voev (2008), Chiriac and Voev (2011) and Bauer and Vorkink (2011). The focus in these studies is on developing parsimonious models to forecast the realized covariance matrix. In contrast, this paper develops a framework for forecasting the covariance of daily returns which also requires forecasts of the realized measure. We find the realized measure to be a more precise factor to drive the volatility dynamics for daily returns compared to the outer product of daily returns which is used in GARCH models.

Jin and Maheu (2010) pursue an objective similar to ours by utilizing realized measures to improve the density forecasts of multivariate daily returns; however, their model is different from ours as it is cast in the multivariate stochastic volatility framework. In addition, they propose a different nexus between the dynamics of daily returns and the realized measure. The implication of this is that our model is much easier to estimate and allows for straightforward out-of-sample model evaluation since we provide closed-form forecasting formula.

The structure of the paper is as follows: Section 2 introduces multivariate HEAVY models with some detailed analysis of their properties using a linear specification. Section 3 discusses estimation and inference. In Section 4, we present the out-of-sample model evaluation framework. Section 5 contains the results of our empirical analysis, while Section 6 concludes the paper. Appendix A includes definitions and results from matrix algebra and matrix calculus which are used in some of the derivations and proofs. Appendix B derives the second moments' structure implied by the model. Appendix $\mathrm{C}$ gives a brief overview of the Wishart distribution which we employ in specifying the density of the innovations. All proofs are collected in Appendix D.

\section{Multivariate HEAVY Models}

\subsection{Definitions and Notation}

Let the multivariate log-price process be given by the $(k \times 1)$ vector $Y_{\tau}^{*}$, where $\tau \in \mathbb{R}_{+}$represent continuous time. Suppose we observe $m+1$ intra-daily prices, assumed to be uniformly spaced, so that the $j^{\text {th }}$ intra-daily vector of returns on day $t$ is given by

$$
R_{j, t}=Y_{(t-1)+\frac{j}{m}}^{*}-Y_{(t-1)+\frac{(j-1)}{m}}^{*}, \quad j=1, \ldots, m, \quad t=1,2, \ldots
$$

Assuming, for instance, 24-hour trading means $m=1440$ and $R_{j, t}$ is the vector of returns for

the $j^{\text {th }}$ minute on day $t$. The vector of daily returns is $R_{t}=\sum_{j=1}^{m} R_{j, t}$. The outer product of daily returns is the $(k \times k)$ matrix denoted by $P_{t}=R_{t} R_{t}^{\prime}$. The realized measure on day $t$ is a $(k \times k)$ 
matrix denoted by $V_{t}$. One example of $V_{t}$ which we use in this paper is the realized covariance $\left(R C_{t}\right)$ matrix defined as

$$
R C_{t}=\sum_{j=1}^{m} R_{j, t} R_{j, t}^{\prime} .
$$

Barndorff-Nielsen and Shephard (2004) show that, in the absence of market microstructure noise, $R C_{t}$ is a mixed normal consistent estimator of the quadratic covariation of $Y_{\tau}^{*}$ as $m \rightarrow \infty$. In the presence of market microstructure noise, $R C_{t}$ is a biased estimator. Therefore, in practice one needs to sample sparsely and use subsampling. An alternative is to use a noise-robust estimator such as the realized kernel of Barndorff-Nielsen et al. (2008, 2011).

Letting $\mathcal{F}_{t}^{L F}$ and $\mathcal{F}_{t}^{H F}$ be as defined previously, the HEAVY model is the 2-equation system

$$
\begin{gathered}
\mathrm{E}\left[P_{t} \mid \mathcal{F}_{t-1}^{H F}\right]=\mathrm{E}\left[R_{t} R_{t}^{\prime} \mid \mathcal{F}_{t-1}^{H F}\right]:=H_{t}, \\
\mathrm{E}\left[V_{t} \mid \mathcal{F}_{t-1}^{H F}\right]:=M_{t},
\end{gathered}
$$

where, for simplicity, we assume $\mathrm{E}\left[R_{t} \mid \mathcal{F}_{t-1}^{H F}\right]=0$ so that $H_{t}$ is the conditional covariance matrix of daily returns, or alternatively, the conditional expectation of the outer product of daily returns. We will occasionally use $\mathrm{E}_{t}[\cdot]:=\mathrm{E}\left[\cdot \mid \mathcal{F}_{t}^{H F}\right]$ to denote the expectation conditional on $\mathcal{F}_{t}^{H F}$. Thus, the two latent conditional first moments $\left(H_{t}, M_{t}\right)$ are assumed $\mathcal{F}_{t-1}^{H F}$-measurable.

We shall call (1) and (2) the HEAVY-P and HEAVY-V equations, respectively. HEAVY models can be equivalently represented as

$$
\begin{gathered}
P_{t}=H_{t}^{\frac{1}{2}} \varepsilon_{t} H_{t}^{\frac{1}{2}}, \\
V_{t}=M_{t}^{\frac{1}{2}} \eta_{t} M_{t}^{\frac{1}{2}},
\end{gathered}
$$

where $\varepsilon_{t}$ and $\eta_{t}$ are $(k \times k)$ symmetric innovation matrices satisfying $\mathrm{E}_{t-1}\left[\varepsilon_{t}\right]=\mathrm{E}_{t-1}\left[\eta_{t}\right]=I_{k}$, where $I_{k}$ is an identity matrix. We have defined the symmetric square root of a generic positive semidefinite matrix $A$, denoted by $A^{\frac{1}{2}}$, using the spectral decomposition such that $A^{\frac{1}{2}}=U \Lambda^{\frac{1}{2}} U^{\prime}$ where $U$ is a matrix containing the eigenvectors of $A$, and $\Lambda^{\frac{1}{2}}$ is a diagonal matrix containing the square root of the eigenvalues of $A$. This representation is a matrix-variate generalization of the univariate MEM introduced in Engle (2002) and the vector MEM presented in Cipollini et al. (2007).

Since our focus is on multivariate volatility models, we use the terms HEAVY and GARCH to refer to their multivariate formulation unless otherwise stated. The difference between the HEAVY$\mathrm{P}$ equation and the GARCH model is the conditioning information set. GARCH models condition on $\mathcal{F}_{t-1}^{L F}$ and thus $H_{t}$ is influenced by the squares and cross products of past daily returns (i.e. lags of $P_{t}$ ). In the HEAVY-P equation, we condition on $\mathcal{F}_{t-1}^{H F}$ which enables us to use lags of $V_{t}$ to project the path of $H_{t}$.

Equations (1) and (2), or equivalently (3) and (4), define a class of models which links the dynamics of $H_{t}$ to the realized measure, $V_{t}$. This becomes clear once we specify the dynamic equations for $H_{t}$ and $M_{t}$, where it is assumed that both are $\mathcal{F}_{t-1}^{H F}$-measurable. Choosing a particular 
specification for the dynamics of $H_{t}$ and $M_{t}$ yields a particular model within the HEAVY class. For ease of presentation, we will focus in the rest of this paper on one particular specification within the HEAVY class which is akin to a multivariate $\operatorname{GARCH}(1,1)$ model, and we shall refer to it simply as the HEAVY model.

\subsection{Model Parameterization}

One of the main challenges in multivariate volatility modelling is to ensure that the conditional covariance matrix is positive semidefinite. In the GARCH literature, this task was approached using the BEKK parameterization introduced by Engle and Kroner (1995). We can adopt that approach to our model, which we call BEKK-type parameterization although the models are distinct. The BEKK-type parameterization is

$$
\begin{gathered}
H_{t}=\bar{C}_{H} \bar{C}_{H}^{\prime}+\bar{B}_{H} H_{t-1} \bar{B}_{H}^{\prime}+\bar{A}_{H} V_{t-1} \bar{A}_{H}^{\prime}, \\
M_{t}=\bar{C}_{M} \bar{C}_{M}^{\prime}+\bar{B}_{M} M_{t-1} \bar{B}_{M}^{\prime}+\bar{A}_{M} V_{t-1} \bar{A}_{M}^{\prime} .
\end{gathered}
$$

The $(k \times k)$ matrices $\bar{A}_{H}, \bar{B}_{H}, \bar{A}_{M}$ and $\bar{B}_{M}$ each have $k^{2}$ free parameters, while $\bar{C}_{H}$ and $\bar{C}_{M}$ are $(k \times k)$ lower triangular matrices each with $k^{*}=k(k+1) / 2$ free parameters. The parameterization in (5) and (6) guarantees that $H_{t}$ and $M_{t}$ are positive semidefinite for all $t$ assuming $H_{0}$ and $M_{0}$ are positive semidefinite. If, in addition, $\bar{C}_{H}$ and $\bar{C}_{M}$ are full rank matrices, then $H_{t}$ and $M_{t}$ are positive definite for all $t$. We refer to $\bar{A}_{H}, \bar{B}_{H}, \bar{A}_{M}$ and $\bar{B}_{M}$ as the dynamic parameters, which are of main interest to us. Sometimes we consider $\bar{C}_{H}$ and $\bar{C}_{M}$ to be "nuisance parameters".

Remark 1 Although our interest is to obtain multi-step forecasts of $H_{t}$, forecasts from (6) are needed due to the presence of $V_{t-1}$ in (5). Forecasting the realized measure itself has been the focus of a number of recent studies (e.g. Andersen et al. (2003, 2007, 2011)). We note that postulating GARCH-type dynamics for the realized measure is consistent with its empirical properties such as time-varying volatility of realized volatility and evidence of excess kurtosis; see Corsi et al. (2008). Therefore, (6) may produce accurate forecasts of $M_{t}$.

The unrestricted BEKK-type parameterization in (5) and (6) has $O\left(k^{2}\right)$ parameters. To avoid the curse of dimensionality one could impose that $\bar{A}_{H}, \bar{B}_{H}, \bar{A}_{M}$ and $\bar{B}_{M}$ are scalars or diagonal matrices, which yields the scalar or diagonal HEAVY model, respectively. In either case, the resulting equations for the diagonal elements of $H_{t}$ and $M_{t}$ would constitute univariate HEAVY models. The equations for the off-diagonal elements would also have a HEAVY structure in which the conditional covariances are driven by their own lags and the corresponding realized covariances. If the elements of $\bar{A}_{H}, \bar{B}_{H}, \bar{A}_{M}$ and $\bar{B}_{M}$ are unrestricted (i.e. a full HEAVY parameterization), the multivariate HEAVY model no longer comprises univariate HEAVY models, since in this case the evolution of every element in $H_{t}$ and $M_{t}$ will be influenced by own as well as cross-asset effects. 
Example 1 Let $a_{i j}$ denote the $(i, j)^{\text {th }}$ element of any matrix $A$. The $H_{t}$ equation in a bivariate diagonal HEAVY model is given by

$$
\begin{aligned}
\left(\begin{array}{ll}
h_{11, t} & h_{12, t} \\
h_{21, t} & h_{22, t}
\end{array}\right)= & \left(\begin{array}{cc}
\bar{c}_{11, H} & 0 \\
\bar{c}_{21, H} & \bar{c}_{22, H}
\end{array}\right)\left(\begin{array}{cc}
\bar{c}_{11, H} & 0 \\
\bar{c}_{21, H} & \bar{c}_{22, H}
\end{array}\right)^{\prime} \\
& +\left(\begin{array}{cc}
\bar{b}_{11, H} & 0 \\
0 & \bar{b}_{22, H}
\end{array}\right)\left(\begin{array}{ll}
h_{11, t-1} & h_{12, t-1} \\
h_{21, t-1} & h_{22, t-1}
\end{array}\right)\left(\begin{array}{cc}
\bar{b}_{11, H} & 0 \\
0 & \bar{b}_{22, H}
\end{array}\right) \\
& +\left(\begin{array}{cc}
\bar{a}_{11, H} & 0 \\
0 & \bar{a}_{22, H}
\end{array}\right)\left(\begin{array}{ll}
v_{11, t-1} & v_{12, t-1} \\
v_{21, t-1} & v_{22, t-1}
\end{array}\right)\left(\begin{array}{cc}
\bar{a}_{11, H} & 0 \\
0 & \bar{a}_{22, H}
\end{array}\right) .
\end{aligned}
$$

To better understand the dynamics, we express (5) and (6) in vector form. ${ }^{2}$ Define $p_{t}:=$ $\operatorname{vech}\left(P_{t}\right), v_{t}:=\operatorname{vech}\left(V_{t}\right), h_{t}:=\operatorname{vech}\left(H_{t}\right)$ and $m_{t}:=\operatorname{vech}\left(M_{t}\right)$, where the vech operator stacks the lower triangular part including the main diagonal of a $(k \times k)$ symmetric matrix into a $\left(k^{*} \times 1\right)$ vector, $k^{*}=k(k+1) / 2$. These $\left(k^{*} \times 1\right)$ vectors retain the unique elements of the matrices of interest to us. An equivalent representation of (3) and (4) is

$$
P_{t}=H_{t}+H_{t}^{\frac{1}{2}}\left(\varepsilon_{t}-I_{k}\right) H_{t}^{\frac{1}{2}}, \quad V_{t}=M_{t}+M_{t}^{\frac{1}{2}}\left(\eta_{t}-I_{k}\right) M_{t}^{\frac{1}{2}},
$$

which, using the vech notation, can be expressed as

$$
p_{t}=h_{t}+\widetilde{\varepsilon}_{t}, \quad v_{t}=m_{t}+\widetilde{\eta}_{t}
$$

where $\widetilde{\varepsilon}_{t}=\operatorname{vech}\left(H_{t}^{\frac{1}{2}}\left(\varepsilon_{t}-I_{k}\right) H_{t}^{\frac{1}{2}}\right)=L_{k}\left(H_{t}^{\frac{1}{2}} \otimes H_{t}^{\frac{1}{2}}\right) D_{k} \operatorname{vech}\left(\varepsilon_{t}-I_{k}\right)$ and $\widetilde{\eta}_{t}=\operatorname{vech}\left(M_{t}^{\frac{1}{2}}\left(\eta_{t}-I_{k}\right) M_{t}^{\frac{1}{2}}\right)=$ $L_{k}\left(M_{t}^{\frac{1}{2}} \otimes M_{t}^{\frac{1}{2}}\right) D_{k} v e c h\left(\eta_{t}-I_{k}\right)$. The second equality in each expression follows from (A.1), and $L_{k}$ and $D_{k}$ are, respectively, the elimination and duplication matrices defined in Appendix A. This representation is particularly convenient since $\widetilde{\varepsilon}_{t}$ and $\widetilde{\eta}_{t}$ are vector martingale difference sequence with respect to $\mathcal{F}_{t-1}^{H F}$.

Similarly, (5) and (6) can be written as

$$
\begin{gathered}
h_{t}=C_{H}+B_{H} h_{t-1}+A_{H} v_{t-1}, \\
m_{t}=C_{M}+B_{M} m_{t-1}+A_{M} v_{t-1},
\end{gathered}
$$

where $C_{H}=L_{k}\left(\bar{C}_{H} \otimes \bar{C}_{H}\right) D_{k} \operatorname{vech}\left(I_{k}\right), B_{H}=L_{k}\left(\bar{B}_{H} \otimes \bar{B}_{H}\right) D_{k}$ and $A_{H}=L_{k}\left(\bar{A}_{H} \otimes \bar{A}_{H}\right) D_{k}$, where we make use of (A.1). $C_{M}, B_{M}$, and $A_{M}$ are defined similarly using the parameters of (6). $C_{H}$ and $C_{M}$ are $\left(k^{*} \times 1\right)$ vectors, while $A_{H}, B_{H}, A_{M}$ and $B_{M}$ are $\left(k^{*} \times k^{*}\right)$ matrices. The elimination and duplication matrices, $L_{k}$ and $D_{k}$, are non-stochastic matrices of zeros and ones, so the parameters in (7)-(8) are uniquely identified from (5)-(6) and vice versa.

\footnotetext{
${ }^{2}$ We use matrix results which are collected in Appendix A for ease of reference.
} 
Example 2 The vech representation of the $H_{t}$ equation of the bivariate diagonal HEAVY model in Example 1 is

$$
\begin{aligned}
\left(\begin{array}{l}
h_{11, t} \\
h_{21, t} \\
h_{22, t}
\end{array}\right)= & \left(\begin{array}{c}
\bar{c}_{11, H}^{2} \\
\bar{c}_{11, H} \bar{c}_{21, H} \\
\bar{c}_{21, H}^{2}+\bar{c}_{22, H}^{2}
\end{array}\right)+\left(\begin{array}{ccc}
\bar{b}_{11, H}^{2} & 0 & 0 \\
0 & \bar{b}_{11, H} \bar{b}_{22, H} & 0 \\
0 & 0 & \bar{b}_{22, H}^{2}
\end{array}\right)\left(\begin{array}{l}
h_{11, t-1} \\
h_{21, t-1} \\
h_{22, t-1}
\end{array}\right) \\
& +\left(\begin{array}{ccc}
\bar{a}_{11, H}^{2} & 0 & 0 \\
0 & \bar{a}_{11, H} \bar{a}_{22, H} & 0 \\
0 & 0 & \bar{a}_{22, H}^{2}
\end{array}\right)\left(\begin{array}{l}
v_{11, t-1} \\
v_{21, t-1} \\
v_{22, t-1}
\end{array}\right) .
\end{aligned}
$$

By substituting $h_{t}=p_{t}-\widetilde{\varepsilon}_{t}$ and $m_{t}=v_{t}-\widetilde{\eta}_{t}$ into (7) and (8), it is straightforward to show that the HEAVY model has the following VARMA(1,1) representation

$$
\left(\begin{array}{c}
p_{t} \\
v_{t}
\end{array}\right)=\left(\begin{array}{c}
C_{H} \\
C_{M}
\end{array}\right)+\left(\begin{array}{cc}
B_{H} & A_{H} \\
0 & B_{M}+A_{M}
\end{array}\right)\left(\begin{array}{c}
p_{t-1} \\
v_{t-1}
\end{array}\right)+\left(\begin{array}{c}
\widetilde{\varepsilon}_{t} \\
\widetilde{\eta}_{t}
\end{array}\right)-\left(\begin{array}{cc}
B_{H} & 0 \\
0 & B_{M}
\end{array}\right)\left(\begin{array}{c}
\widetilde{\varepsilon}_{t-1} \\
\widetilde{\eta}_{t-1}
\end{array}\right)
$$

since $\left(\widetilde{\varepsilon}_{t}^{\prime}, \widetilde{\eta}_{t}^{\prime}\right)^{\prime}$ is a vector martingale difference sequence with respect to $\mathcal{F}_{t-1}^{H F}$, assuming $\operatorname{Var}\left[\left(\widetilde{\varepsilon}_{t}^{\prime}, \widetilde{\eta}_{t}^{\prime}\right)^{\prime}\right]$ exists. The coefficient matrix attached to $\left(p_{t-1}^{\prime}, v_{t-1}^{\prime}\right)^{\prime}$ determines the persistence of the HEAVY system. For covariance stationarity, the eigenvalues of this matrix must be less than one in modulus. Since it is block triangular, its eigenvalues are members of the multiset of the eigenvalues of $B_{H}$ and $\left(B_{M}+A_{M}\right) .{ }^{3}$ In the following assumption we explicitly state this covariance stationarity condition, where for any $(k \times k)$ matrix $A$ with eigenvalues $\lambda_{1}, \ldots, \lambda_{k}, \rho(A):=\max _{i}\left|\lambda_{i}\right|$ denotes the spectral radius of $A$.

Assumption 1 In the HEAVY model given by (7) and (8), $\rho\left(B_{H}\right)<1$ and $\rho\left(B_{M}+A_{M}\right)<1$.

Remark 2 The covariance stationarity condition in Assumption 1 is analogous to the one given in Engle and Kroner (1995). This can be seen by noting that for any square matrix $A, D_{k}^{+}(A \otimes A) D_{k}$ and $(A \otimes A)$ have the same eigenvalues by Magnus and Neudecker (1999, Theorem 1, Chapter 2) and Magnus (1988, Theorem 4.10), where $D_{k}^{+}=\left(D_{k}^{\prime} D_{k}\right)^{-1} D_{k}^{\prime}$ is the Moore-Penrose inverse of $D_{k}$. Also, it holds that for any square matrix $A, D_{k}^{+}(A \otimes A) D_{k}=L_{k}(A \otimes A) D_{k}$; see Lutkepohl (1996, Section 9.5.5). Thus, $B_{H}=L_{k}\left(\bar{B}_{H} \otimes \bar{B}_{H}\right) D_{k}$ and $\left(\bar{B}_{H} \otimes \bar{B}_{H}\right)$ have the same eigenvalues. A similar argument applies to $\left(B_{M}+A_{M}\right)$.

\subsection{Covariance Targeting}

The covariance targeting parameterization was introduced by Engle and Mezrich (1996) for the univariate GARCH model. This allows the unconditional moments of the model to be estimated by the empirical moments, and means its forecast tends to the unconditional moments as the forecast

\footnotetext{
${ }^{3} \mathrm{~A}$ multiset is a set that allows for some or all of its elements to be repeated. This general definition is needed to allow for the case when $B_{H}$ and $\left(B_{M}+A_{M}\right)$ have some common eigenvalues.
} 
horizon tends to infinity. The dynamic parameters would then be estimated using a quasi-likelihood. Here we discuss unique issues related to restricted models and derive the covariance targeting parameterization of the HEAVY model, which is not immediately straightforward. The multivariate HEAVY model differs from previous ARCH-type models by using a shock other than the outerproduct of returns to model the conditional covariance. This has an unintended consequence of changing the interpretation of the estimated parameters when the dynamics of the model are restricted from the full specification in (5), as is the case when $\bar{A}_{H}$ is assumed to be diagonal or scalar.

Let $\Omega_{H}:=\mathrm{E}\left[P_{t}\right]=\mathrm{E}\left[H_{t}\right]$ and $\Omega_{M}:=\mathrm{E}\left[V_{t}\right]=\mathrm{E}\left[M_{t}\right] .{ }^{4}$ Taking unconditional expectation of (5) and (6) gives

$$
\Omega_{H}=\bar{C}_{H} \bar{C}_{H}^{\prime}+\bar{B}_{H} \Omega_{H} \bar{B}_{H}^{\prime}+\bar{A}_{H} \Omega_{M} \bar{A}_{H}^{\prime}, \quad \Omega_{M}=\bar{C}_{M} \bar{C}_{M}^{\prime}+\bar{B}_{M} \Omega_{M} \bar{B}_{M}^{\prime}+\bar{A}_{M} \Omega_{M} \bar{A}_{M}^{\prime} .
$$

Define $\bar{\kappa}=\Omega_{M}^{\frac{1}{2}} \Omega_{H}^{-\frac{1}{2}}$ as a useful expression relating $\Omega_{H}$ to $\Omega_{M}$. It is worth noting that although $\Omega_{M}^{\frac{1}{2}}$ and $\Omega_{H}^{-\frac{1}{2}}$ are both symmetric matrices, $\bar{\kappa}$ is not necessarily symmetric. Consider the case when $\bar{\kappa} \neq I_{k}$ so that the unconditional expectation of the realized measure is not equal to the unconditional expectation of the outer-product of returns. In this case it may be tempting to redefine the HEAVY-P equation (5) in terms of the rotated realized measure $\widetilde{V}_{t-1}=\bar{\kappa}^{-1} V_{t-1}\left(\bar{\kappa}^{-1}\right)^{\prime}$. The unrestricted HEAVY-P equation is invariant to this transformation since

$$
\begin{aligned}
H_{t} & =\bar{C}_{H} \bar{C}_{H}^{\prime}+\bar{B}_{H} H_{t-1} \bar{B}_{H}^{\prime}+\bar{A}_{H} V_{t-1} \bar{A}_{H}^{\prime} \\
& =\bar{C}_{H} \bar{C}_{H}^{\prime}+\bar{B}_{H} H_{t-1} \bar{B}_{H}^{\prime}+\left(\bar{A}_{H} \bar{\kappa}\right) \bar{\kappa}^{-1} V_{t-1}\left(\bar{\kappa}^{-1}\right)^{\prime}\left(\bar{A}_{H} \bar{\kappa}\right)^{\prime} \\
& =\bar{C}_{H} \bar{C}_{H}^{\prime}+\bar{B}_{H} H_{t-1} \bar{B}_{H}^{\prime}+\bar{A}_{H}^{*} \widetilde{V}_{t-1} \bar{A}_{H}^{* \prime},
\end{aligned}
$$

where $\bar{A}_{H}^{*}=\bar{A}_{H} \bar{\kappa}$. However, this invariance does not hold when $\bar{A}_{H}$ is assumed to be diagonal or scalar. For example, suppose $\bar{A}_{H}$ is restricted to be scalar (i.e. $\bar{A}_{H}=\bar{\alpha}_{H} I_{k}$, where $\bar{\alpha}_{H}$ is scalar) and so all series have common loadings on the realized measure. The only case where a model driven by the rotated realized measure, $\widetilde{V}_{t-1}$, will correspond to a model driven by the unrotated realized measure, $V_{t-1}$, occurs when $\bar{\kappa}$ is diagonal with a single, common value in all non-zero locations. The two parameterizations of the HEAVY-P equation will only produce the same fit if $\bar{\alpha}_{H}^{*}=\bar{\alpha}_{H} \bar{\kappa}$, which is only possible if $\bar{\kappa} \propto I_{k}$. The diagonal case is similar, only that the set of models where the two parameterizations are equivalent occurs when $\bar{\kappa}$ is diagonal.

These two model specifications lead to two different methods to covariance target. Letting $\omega_{H}$ and $\omega_{M}$ denote $\operatorname{vech}\left(\Omega_{H}\right)$ and $\operatorname{vech}\left(\Omega_{M}\right)$, respectively, the following proposition gives two covariance targeting parameterizations of the HEAVY model.

\footnotetext{
${ }^{4}$ Since our model is formulated for the squares and cross-products of daily returns (elements of $P_{t}$ ) and the realized variances and covariances (elements of $V_{t}$ ), the elements of $\Omega_{H}$ are the unconditional variances and covariances of daily returns, while the elements of $\Omega_{M}$ denote the unconditional expectation of realized variances and covariances.
} 
Proposition 1 The covariance targeting parameterization of the HEAVY model in (7) and (8) is

$$
\begin{aligned}
h_{t} & =\left(I_{k^{*}}-B_{H}-A_{H} \kappa\right) \omega_{H}+B_{H} h_{t-1}+A_{H} v_{t-1}, \\
m_{t} & =\left(I_{k^{*}}-B_{M}-A_{M}\right) \omega_{M}+B_{M} m_{t-1}+A_{M} v_{t-1},
\end{aligned}
$$

where $\kappa=L_{k}(\bar{\kappa} \otimes \bar{\kappa}) D_{k}, \bar{\kappa}=\Omega_{M}^{\frac{1}{2}} \Omega_{H}^{-\frac{1}{2}}, \omega_{H}:=\operatorname{vech}\left(\Omega_{H}\right), \omega_{M}:=\operatorname{vech}\left(\Omega_{M}\right)$, and $L_{k}$ and $D_{k}$ denote respectively the elimination and duplication matrices of order $k$. An alternative covariance targeting parameterization for (7) is

$$
h_{t}=\left(I_{k^{*}}-B_{H}-A_{H}^{*}\right) \omega_{H}+B_{H} h_{t-1}+A_{H}^{*} \widetilde{v}_{t-1},
$$

where $\widetilde{v}_{t}=\kappa^{-1} v_{t}$ is a rotated realized measure such that $\mathrm{E}\left[\widetilde{v}_{t}\right]=\omega_{H}$.

Remark 3 While the covariance targeting specification in (9)-(10) is a reparameterization of the original model in (7)-(8), the specification (11)-(10) corresponds to a different model which uses a rotated rather than the original realized measure. This is why the coefficient matrix on $\widetilde{v}_{t-1}$ is now denoted by $A_{H}^{*}$. The two models coincide, implying $A_{H}^{*}=A_{H} \kappa$ holds, if and only if both $A_{H}^{*}$ and $A_{H}$ are fully parameterized matrices as discussed above. Using (11)-(10) has the advantage that it is easier to impose the condition $\rho\left(B_{H}+A_{H}^{*}\right)<1$ during estimation; see Assumption 2 below. Imposing the condition $\rho\left(B_{H}+A_{H} \kappa\right)<1$ is more involved, particularly in the diagonal and full HEAVY models since $\kappa$ is a $\left(k^{*} \times k^{*}\right)$ matrix with non-zero elements.

For the covariance targeting parameterization to be sensible, we need (9)-(10), or alternatively (11)-(10), to be consistent with a positive definite long run target for $H_{t}$ and $M_{t}$. Therefore, we replace Assumption 1 with the following assumption which guarantees both covariance stationarity of $h_{t}$ and $m_{t}$ as well as having positive definite targets.

Assumption 2 In the covariance targeting parameterization of the HEAVY model given by (9)(10), $\rho\left(B_{H}+A_{H} \kappa\right)<1$ and $\rho\left(B_{M}+A_{M}\right)<1$. In the covariance targeting parameterization of the HEAVY model given by (11)-(10), $\rho\left(B_{H}+A_{H}^{*}\right)<1$ and $\rho\left(B_{M}+A_{M}\right)<1$.

Estimating the model in its covariance targeting specification given by (9)-(10) can be carried out in two steps. In the first step, we replace $\Omega_{H}$ and $\Omega_{M}$ with their moment estimators,

$$
\widehat{\Omega}_{H}=T^{-1} \sum_{t=1}^{T} P_{t}, \quad \widehat{\Omega}_{M}=T^{-1} \sum_{t=1}^{T} V_{t},
$$

which also gives the estimates $\widehat{\bar{\kappa}}=\widehat{\Omega}_{M}^{\frac{1}{2}} \widehat{\Omega}_{H}^{-\frac{1}{2}}$ and $\widehat{\kappa}=L_{k}(\widehat{\bar{\kappa}} \otimes \widehat{\bar{\kappa}}) D_{k}$. In the second step, we estimate the dynamic parameters $\left(A_{H}, B_{H}, A_{M}\right.$ and $\left.B_{M}\right)$ given $\widehat{\kappa}$. Alternatively, if the covariance targeting specification is given by (11)-(10), the estimate $\widehat{\kappa}$ is used to compute the rotated realized measure $\widetilde{v}_{t}=\widehat{\kappa}^{-1} v_{t}$, which is then used in (11) replacing the original realized measure. 
We can also express the unconditional moments in terms of the model parameters. By taking unconditional expectation of (7) and (8), it is straightforward to show that the unconditional expectation of $p_{t}$ and $v_{t}$ is given by

$$
\begin{gathered}
\omega_{H}=\left(I_{k^{*}}-B_{H}\right)^{-1}\left[C_{H}+A_{H}\left(I_{k^{*}}-B_{M}-A_{M}\right)^{-1} C_{M}\right], \\
\omega_{M}=\left(I_{k^{*}}-B_{M}-A_{M}\right)^{-1} C_{M},
\end{gathered}
$$

where we substituted for $\omega_{M}$ into the equation for $\omega_{H}$. Equations (12) and (13) allow for computing the long-run covariance matrices implied by a set of parameter estimates when the model is not in its covariance targeting specification, and the expression in (13) is analogous to that of GARCH processes. In Appendix B, we derive the unconditional second moments of $p_{t}$ and $v_{t}$, which correspond to the fourth moments of the returns (i.e. kurtosis) and second moments of the realized measure (i.e. volatility of volatility).

\subsection{Multi-Step Forecasting}

We are primarily interested in forecasting the conditional covariance of daily returns, $H_{t}$. One-step forecasts are directly computable using (7), which expresses $H_{t}$ in its vech form. To compute $s$-step forecasts for $s=2,3, \ldots$, we need the forecasts from (8) as well to compute the $s$-step conditional expectation of the realized measure appearing in the right-hand-side of (7).

Given the intact structure of the model, we are able to derive a closed-form expression for the $s$-step forecast of $H_{t}$, that is $\mathrm{E}\left[H_{t+s} \mid \mathcal{F}_{t}^{H F}\right]$, or equivalently, $\mathrm{E}\left[h_{t+s} \mid \mathcal{F}_{t}^{H F}\right]$. This is given in the following proposition.

Proposition 2 Let the model be given by (7) and (8), then the s-step forecast of $h_{t}$ is

$$
\begin{aligned}
\mathrm{E}_{t}\left[h_{t+s}\right]= & \sum_{i=1}^{s-1} B_{H}^{i-1} C_{H}+B_{H}^{s-1} h_{t+1} \\
& +\sum_{i=1}^{s-1} B_{H}^{i-1} A_{H}\left\{\sum_{j=1}^{s-i-1}\left(B_{M}+A_{M}\right)^{j-1} C_{M}+\left(B_{M}+A_{M}\right)^{s-i-1} m_{t+1}\right\}
\end{aligned}
$$

where $h_{t+1}$ and $m_{t+1}$ are $\mathcal{F}_{t}^{H F}$-measurable. Alternatively, let the model be given by (9) and (10), then the s-step forecast of $h_{t}$ is

$$
\mathrm{E}_{t}\left[h_{t+s}\right]=\omega_{H}+B_{H}^{s-1}\left(h_{t+1}-\omega_{H}\right)+\sum_{i=1}^{s-1} B_{H}^{i-1} A_{H}\left(B_{M}+A_{M}\right)^{s-i-1}\left(m_{t+1}-\omega_{M}\right) .
$$

The difference between (14) and (15) is that the latter is obtained under a covariance targeting specification in which the constant terms $C_{H}$ and $C_{M}$ are replaced with expressions involving $\omega_{H}$ and $\omega_{M}$ as shown in 2.3. In (14), Assumption 1 implies that $\mathrm{E}_{t}\left[h_{t+s}\right] \rightarrow \omega_{H}$ as $s \rightarrow \infty$ since the coefficients on $h_{t+1}$ and $m_{t+1}$ will tend to zero, while the limit of the constant terms including $C_{H}$ 


\begin{tabular}{ccccccc}
\hline & \multicolumn{5}{c}{$\left(B_{M}+A_{M}\right)$} \\
& & 0.900 & 0.950 & 0.990 & 0.995 & 0.999 \\
\cline { 3 - 7 }$B_{H}$ & 0.65 & 6 & 8 & 18 & 31 & 138 \\
& 0.70 & 8 & 11 & 33 & 62 & 292 \\
& 0.75 & 10 & 15 & 52 & 99 & 475 \\
& 0.80 & 13 & 20 & 76 & 145 & 699 \\
& 0.85 & 18 & 28 & 106 & 204 & 989 \\
\hline \multirow{5}{*}{$B_{H}$} & 0.65 & 10 & 15 & 58 & 112 & 543 \\
& 0.70 & 12 & 19 & 74 & 143 & 698 \\
& 0.75 & 14 & 23 & 93 & 180 & 881 \\
& 0.80 & 17 & 28 & 116 & 226 & 1105 \\
& 0.85 & 22 & 36 & 146 & 285 & 1394 \\
\hline
\end{tabular}

Table 1: Covariance targeting scalar HEAVY model half-life (in days) for different parameter values. The figures are approximations to the nearest integer.

and $C_{M}$ will simplify to the right hand side of (12). In (15), we also have that $\mathrm{E}_{t}\left[h_{t+s}\right] \rightarrow \omega_{H}$ as $s \rightarrow \infty$; however, in this case Assumption 2 is the operative assumption since the derivation of this equation is based on the covariance targeting specification.

Remark 4 In deriving (15), we focused on the covariance targeting specification given by (9)-(10) since it is more constructive to think about the persistence of the HEAVY model as discussed below. To forecast using the covariance targeting specification in (11)-(10), $A_{H}^{*}$ will appear in (15) instead of $A_{H}$. Thus, the term $\left(m_{t+1}-\omega_{M}\right)$ must be pre-multiplied by $\kappa^{-1}$ to ensure positive definiteness of the forecast matrix $\mathrm{E}_{t}\left[H_{t+s}\right]$.

A measure of persistence usually used in GARCH models is the half-life of a deviation of the 1-step forecast of $h_{t}$ from its long run, which is the number of days, $s$, it takes for half of this deviation to disappear. This can be computed using (15). Table 1 gives an approximation to the nearest integer of the half-life of the covariance targeting scalar HEAVY model for different parameter values. ${ }^{5}$ Generally, our empirical results indicate that the HEAVY model half-life is substantially shorter than that of the GARCH model suggesting its forecasts respond faster to abrupt changes in the level of volatility or correlation.

The short-run momentum in HEAVY forecasts can also be seen from (15). In GARCH models only the gap $\left(h_{t+1}-\omega_{H}\right)$ appears in the forecast formula which implies monotonic mean reversion to $\omega_{H}$ as the forecast horizon increases. In (15), the presence of the additional gap $\left(m_{t+1}-\omega_{M}\right)$

\footnotetext{
${ }^{5}$ In computing the half-life, we assume that the two gaps, $\left(h_{t+1}-\omega_{H}\right)$ and $\left(m_{t+1}-\omega_{M}\right)$, are of equal size which is set, without loss of generality, equal to a $\left(k^{*} \times 1\right)$ vector of ones. This enables comparison with the GARCH model half-life since its forecast formula contains only the gap $\left(h_{t+1}-\omega_{H}\right)$. Also implicit in our assumption is that both gaps have the same sign which is in line with our empirical finding that $h_{t}$ and $m_{t}$ tend to be very highly correlated.
} 
implies that mean reversion is not necessarily monotonic. We demonstrate this property in our empirical analysis; see Figure 4 and related discussion.

\section{Estimation and Inference}

\subsection{The Distribution of $\varepsilon_{t}$ and $\eta_{t}$}

For the HEAVY model in (3) and (4)

$$
P_{t}=H_{t}^{\frac{1}{2}} \varepsilon_{t} H_{t}^{\frac{1}{2}}, \quad V_{t}=M_{t}^{\frac{1}{2}} \eta_{t} M_{t}^{\frac{1}{2}}
$$

the natural choice for the density of the innovation matrices, $\varepsilon_{t}$ and $\eta_{t}$, is the Wishart distribution. It is an appropriate choice in models where the support of the random variable of interest is restricted to the space of positive semidefinite matrices. ${ }^{6}$ Appendix $\mathrm{C}$ provides an overview of the Wishart distribution including the definitions and notation used in this section.

In GARCH models, the vector of daily returns is usually modelled as $R_{t}=H_{t}^{\frac{1}{2}} \xi_{t}$ with $\xi_{t} \stackrel{i . i . d}{\sim}$. $N\left(0, I_{k}\right)$, which motivates quasi-maximum likelihood estimation (QMLE). The HEAVY-P equation is expressed for the outer product of returns, $P_{t}$, and in this case we have $P_{t}=R_{t} R_{t}^{\prime}=$ $H_{t}^{\frac{1}{2}} \xi_{t} \xi_{t}^{\prime}\left(H_{t}^{\frac{1}{2}}\right)^{\prime}=H_{t}^{\frac{1}{2}} \varepsilon_{t} H_{t}^{\frac{1}{2}}$, where $\varepsilon_{t}=\xi_{t} \xi_{t}^{\prime}$. The assumption that $\xi_{t} \stackrel{i . i . d .}{\sim} N\left(0, I_{k}\right)$ implies that $\varepsilon_{t}$ follows a Wishart distribution.

One of the key results on Wishart distributions is that if any matrix $S \sim W_{k}(n, \Sigma)$, then $A S A^{\prime} \sim W_{k}\left(n, A \Sigma A^{\prime}\right)$ for any $(k \times k)$ nonsingular matrix $A$. Assuming a Wishart density for $\varepsilon_{t}$ and $\eta_{t}$ implies that $P_{t}$ and $V_{t}$ are assumed to be conditionally Wishart distributed. However, one distinction between the density of $\varepsilon_{t}$ and $\eta_{t}$ relates to the differences in the ranks of $P_{t}$ and $V_{t}$. The matrix $P_{t}=R_{t} R_{t}^{\prime}$ has rank 1 by construction if there is at least one non-zero return in the $R_{t}$ vector. Whether using the realized covariance estimator or the realized kernel of Barndorff-Nielsen et al. (2008), the matrix $V_{t}$ is guaranteed to be full rank under standard regularity conditions. This difference in rank entails that $\varepsilon_{t}$ should have a singular Wishart density and $\eta_{t}$ a standardized Wishart density. The discussion in Appendix $\mathrm{C}$ makes it clear that this distinction is necessary for the two conditional moment assumptions $\mathrm{E}_{t-1}\left[\varepsilon_{t}\right]=I_{k}$ and $\mathrm{E}_{t-1}\left[\eta_{t}\right]=I_{k}$ to be satisfied.

Therefore, we assume $\varepsilon_{t} \stackrel{\text { i.i.d. }}{\sim} S I N G W_{k}\left(1, I_{k}\right)$ and $\eta_{t} \stackrel{\text { i.i.d. }}{\sim} S W_{k}\left(k, I_{k}\right)$. The densities of $\varepsilon_{t}$ and $\eta_{t}$ are given by (C.2) and (C.1), respectively. Thus we have that $P_{t} \mid \mathcal{F}_{t-1}^{H F} \sim S I N G W_{k}\left(1, H_{t}\right)$ and $V_{t} \mid \mathcal{F}_{t-1}^{H F} \sim S W_{k}\left(k, M_{t}\right)$ since $H_{t}$ and $M_{t}$ are $\mathcal{F}_{t-1}^{H F}$-measurable. The distinction between the densities of $\varepsilon_{t}$ and $\eta_{t}$ is of no consequence to QMLE as we show in a moment. However, it is important to have an appropriately specified model satisfying the conditional moment assumptions $\mathrm{E}_{t-1}\left[\varepsilon_{t}\right]=\mathrm{E}_{t-1}\left[\eta_{t}\right]=I_{k}{ }^{7}$

\footnotetext{
${ }^{6}$ Some recent multivariate stochastic volatility models also employ the Wishart distribution to model time-varying correlations; see Chib et al. (2009) and the references cited therein, and also Jin and Maheu (2010).

${ }^{7}$ One can test for the Wishart distributional assumption by making use of the corollary that if $S \sim W_{k}(n, \Sigma)$, then $\frac{a^{\prime} S a}{a^{\prime} \Sigma a} \sim \chi_{(n)}^{2}$ for any $(k \times 1)$ vector $a \neq 0$; see Gupta and Nagar (2000). Also, conditional moment tests can be used
} 


\subsection{Quasi-Maximum Likelihood Estimation}

The HEAVY model is parameterized with a finite-dimensional $(\delta \times 1)$ parameter vector $\theta \in \Theta \subset \mathbb{R}^{\delta}$. Decompose $\theta=\left(\theta_{H}^{\prime}, \theta_{M}^{\prime}\right)^{\prime}$ where the $\left(\delta_{H} \times 1\right)$ vector $\theta_{H}$ and $\left(\delta_{M} \times 1\right)$ vector $\theta_{M}$ denote the parameter vectors of the HEAVY-P and HEAVY-V equations, respectively. Let $\theta_{0}=\left(\theta_{H, 0}^{\prime}, \theta_{M, 0}^{\prime}\right)^{\prime}$ denote the true parameter vector. The log-likelihood for the $t^{\text {th }}$ observation will be denoted by $l_{H, t}\left(\theta_{H}\right)$ and $l_{M, t}\left(\theta_{M}\right)$. Inference for the HEAVY model will be based on QMLE of the following two log-likelihood functions

$$
l_{H, t}\left(\theta_{H}\right)=c_{H}-\frac{1}{2}\left(\log \left|H_{t}\right|+\operatorname{tr}\left(H_{t}^{-1} P_{t}\right)\right), \quad l_{M, t}\left(\theta_{M}\right)=c_{M}-\frac{k}{2}\left(\log \left|M_{t}\right|+\operatorname{tr}\left(M_{t}^{-1} V_{t}\right)\right),
$$

where $c_{H}$ and $c_{M}$ are constants with respect to $\theta_{H}$ and $\theta_{M}$; see (C.2) and (C.1) respectively. Thus the distinction between the densities of $\varepsilon_{t}$ and $\eta_{t}$ is of no consequence for QMLE of the model parameters. Engle and Gallo (2006) argue similarly for the Gamma density where the shape parameter is of no consequence when estimating the scale parameter by QMLE.

We assume the initial values, $H_{0}$ and $M_{0}$, are known and are positive semidefinite. We also assume that $\theta_{H}$ and $\theta_{M}$ are variation free in the sense of Engle et al. (1983), which allows for equation-by-equation estimation. This assumption is not essential and is only used to simplify estimation and inference. The QML estimator is $\widehat{\theta}=\left(\widehat{\theta}_{H}^{\prime}, \widehat{\theta}_{M}^{\prime}\right)^{\prime}$ where

$$
\widehat{\theta}_{H}=\underset{\theta_{H} \in \Theta}{\arg \max } L_{H, t}\left(\theta_{H}\right), \quad \widehat{\theta}_{M}=\underset{\theta_{M} \in \Theta}{\arg \max } L_{M, t}\left(\theta_{M}\right),
$$

and $L_{H, t}\left(\theta_{H}\right)=\sum_{t=1}^{T} l_{H, t}\left(\theta_{H}\right), L_{M, t}\left(\theta_{M}\right)=\sum_{t=1}^{T} l_{M, t}\left(\theta_{M}\right)$.

For the BEKK model, Comte and Lieberman (2003) show strong consistency of QMLE by verifying the conditions given in Jeantheau (1998). Hafner and Preminger (2009) show similar results for the VEC model which nests the BEKK model, and their results also apply to integrated processes. An important condition to establish strong consistency results is for the model to admit a strictly stationary and ergodic solution, which we assume for the HEAVY model.

Before discussing the asymptotic distribution of $\widehat{\theta}$, we first give results on the score vector in the following proposition. It will be convenient to consider the score for each equation separately.

Proposition 3 (i) The score vectors, $S_{H, t}\left(\theta_{H}\right)=\frac{\partial l_{H, t}\left(\theta_{H}\right)}{\partial \theta_{H}^{\prime}}$ and $S_{M, t}\left(\theta_{M}\right)=\frac{\partial l_{M, t}\left(\theta_{M}\right)}{\partial \theta_{M}^{\prime}}$ of dimensions $\left(1 \times \delta_{H}\right)$ and $\left(1 \times \delta_{M}\right)$, respectively, are given by

$$
\begin{gathered}
S_{H, t}\left(\theta_{H}\right)=\frac{\partial l_{H, t}\left(\theta_{H}\right)}{\partial \theta_{H}^{\prime}}=\frac{1}{2}\left[\left(\operatorname{vec}\left(P_{t}\right)\right)^{\prime}-\left(\operatorname{vec}\left(H_{t}\right)\right)^{\prime}\right]\left(H_{t}^{-1} \otimes H_{t}^{-1}\right) \frac{\partial \operatorname{vec}\left(H_{t}\right)}{\partial \theta_{H}^{\prime}}, \\
S_{M, t}\left(\theta_{M}\right)=\frac{\partial l_{M, t}\left(\theta_{M}\right)}{\partial \theta_{M}^{\prime}}=\frac{1}{2}\left[\left(\operatorname{vec}\left(V_{t}\right)\right)^{\prime}-\left(\operatorname{vec}\left(M_{t}\right)\right)^{\prime}\right]\left(M_{t}^{-1} \otimes M_{t}^{-1}\right) \frac{\partial \operatorname{vec}\left(M_{t}\right)}{\partial \theta_{M}^{\prime}} .
\end{gathered}
$$

(ii) Under $\mathrm{E}_{t-1}\left[\varepsilon_{t}\right]=I_{k}$ and $\mathrm{E}_{t-1}\left[\eta_{t}\right]=I_{k}$, the score vectors evaluated at the true parameter values are martingale difference sequence with respect to $\mathcal{F}_{t-1}^{H F}$.

to detect misspecification. 
Remark 5 The scores have a similar structure to those of GARCH models (e.g. Bollerslev and Wooldridge (1992)). In analogy with generalized least squares, the terms in square brackets can be considered "errors", while $\left(H_{t}^{-1} \otimes H_{t}^{-1}\right)$ and $\left(M_{t}^{-1} \otimes M_{t}^{-1}\right)$ are weights and the derivatives $\frac{\partial v e c\left(H_{t}\right)}{\partial \theta_{H}^{\prime}}$ and $\frac{\partial v e c\left(M_{t}\right)}{\partial \theta_{M}^{\prime}}$ are instruments which are orthogonal to the errors at the maximum likelihood estimator, which is a condition for consistency.

To discuss the asymptotic distribution of the QML estimator, $\widehat{\theta}$, we define the $(1 \times \delta)$ combined score vector $S_{t}(\theta)=\left(S_{H, t}\left(\theta_{H}\right), S_{M, t}\left(\theta_{M}\right)\right)$. Having established that the scores are martingale difference sequence with respect to $\mathcal{F}_{t-1}^{H F}$, it can be shown under certain regularity conditions (e.g. Comte and Lieberman (2003)) that

$$
\sqrt{T}\left(\widehat{\theta}-\theta_{0}\right) \stackrel{d}{\longrightarrow} N\left(0, \mathcal{I}^{-1} \mathcal{J I}^{-1}\right)
$$

where

$$
\begin{gathered}
\mathcal{J}=\mathrm{E}\left[S_{t}(\theta)^{\prime} S_{t}(\theta)\right]=\mathrm{E}\left[\begin{array}{cc}
\frac{\partial l_{H, t}\left(\theta_{H}\right)}{\partial \theta_{H}} \frac{\partial l_{H, t}\left(\theta_{H}\right)}{\partial \theta_{H}^{\prime}} & \frac{\partial l_{H, t}\left(\theta_{H}\right)}{\partial \theta_{H}} \frac{\partial l_{M, t}\left(\theta_{M}\right)}{\partial \theta_{M}^{\prime}} \\
\frac{\partial l_{M, t}\left(\theta_{M}\right)}{\partial \theta_{M}} \frac{\partial l_{H, t}\left(\theta_{H}\right)}{\partial \theta_{H}^{\prime}} & \frac{\partial l_{M, t}\left(\theta_{M}\right)}{\partial \theta_{M}} \frac{\partial l_{M, t}\left(\theta_{M}\right)}{\partial \theta_{M}^{\prime}}
\end{array}\right], \\
\mathcal{I}=-\mathrm{E}\left[\frac{\partial S_{t}(\theta)}{\partial \theta}\right]=-\mathrm{E}\left[\begin{array}{cc}
\frac{\partial^{2} l_{H, t}\left(\theta_{H}\right)}{\partial \theta_{H} \partial \theta_{H}^{\prime}} & 0 \\
0 & \frac{\partial^{2} l_{M, t}\left(\theta_{M}\right)}{\partial \theta_{M} \partial \theta_{M}^{\prime}}
\end{array}\right] .
\end{gathered}
$$

The block diagonality of the Hessian, $\mathcal{I}$, is due to the assumption that $\theta_{H}$ and $\theta_{M}$ are variation free, which implies that equation-by-equation standard errors are correct for the HEAVY system. With covariance targeting, a two-step estimation procedure is adopted and in this case the score vector will no longer be a martingale difference sequence, but it will still have mean zero at the true parameter value. Also, the Hessian will be no longer block diagonal due to accounting for the accumulation of estimation error from the first step. We formalize inference in the case of covariance targeting in the following subsection.

\subsection{Two-Step Estimation Under Covariance Targeting}

Under the covariance targeting specification in (9) and (10), the parameter vectors $\theta_{H}$ and $\theta_{M}$ are decomposed into $\theta_{H}=\left(\omega_{H}^{\prime}, \widetilde{\theta}_{H}^{\prime}\right)^{\prime}$ and $\theta_{M}=\left(\omega_{M}^{\prime}, \widetilde{\theta}_{M}^{\prime}\right)^{\prime}$ and are to be estimated in two steps. The unconditional moments, $\omega_{H}$ and $\omega_{M}$, will be estimated in the first step by a moment estimator

$$
\widehat{\omega}_{H}=T^{-1} \sum_{t=1}^{T} p_{t}, \quad \widehat{\omega}_{M}=T^{-1} \sum_{t=1}^{T} v_{t},
$$

and then $\widetilde{\theta}_{H}$ and $\widetilde{\theta}_{M}$ will be estimated by QMLE in the second step. The asymptotics of the QML estimator in this two-step procedure is a direct application of two-step GMM estimation discussed in Newey and McFadden (1994). Define $\widetilde{l}_{H, t}\left(\omega_{H}, \omega_{M}, \widetilde{\theta}_{H}\right)$ and $\widetilde{l}_{M, t}\left(\omega_{M}, \widetilde{\theta}_{M}\right)$ to be the 
$t^{t h}$ observation log-likelihoods for the covariance targeting HEAVY model given by (9) and (10). Two-step estimation gives the following $(1 \times \delta)$ vector of moment conditions

$$
\widetilde{S}_{t}(\widetilde{\theta})=\left(\left(p_{t}-\omega_{H}\right)^{\prime}, \frac{\partial \widetilde{l}_{H, t}}{\partial \widetilde{\theta}_{H}^{\prime}},\left(v_{t}-\omega_{M}\right)^{\prime}, \frac{\partial \widetilde{l}_{M, t}}{\partial \widetilde{\theta}_{M}^{\prime}}\right), \quad \widetilde{\theta}=\left(\omega_{H}^{\prime}, \widetilde{\theta}_{H}^{\prime}, \omega_{M}^{\prime}, \widetilde{\theta}_{M}^{\prime}\right)^{\prime},
$$

which is no longer martingale difference sequence with respect to $\mathcal{F}_{t-1}^{H F}$. In this case

$$
\sqrt{T}\left(\widehat{\theta}-\theta_{0}\right) \stackrel{d}{\longrightarrow} N\left(0, \mathcal{I}^{-1} \mathcal{J}\left(\mathcal{I}^{-1}\right)^{\prime}\right)
$$

where

$$
\begin{gathered}
\mathcal{J}=\operatorname{Var}\left[\frac{1}{\sqrt{T}} \sum_{t=1}^{T} \widetilde{S}_{t}(\widetilde{\theta})\right], \\
\mathcal{I}=-\mathrm{E}\left[\frac{\partial \widetilde{S}_{t}(\widetilde{\theta})}{\partial \widetilde{\theta}}\right]=-\mathrm{E}\left[\begin{array}{cccc}
-I_{k^{*}} & \frac{\partial^{2} \widetilde{l}_{H, t}}{\partial \omega_{H} \partial \widetilde{\theta}_{H}^{\prime}} & 0 & 0 \\
0 & \frac{\partial^{2} \widetilde{l}_{H, t}}{\partial \widetilde{\theta}_{H} \partial \widetilde{\theta}_{H}^{\prime}} & 0 & 0 \\
0 & \frac{\partial^{2} \widetilde{l}_{H, t}}{\partial \omega_{M} \partial \widetilde{\theta}_{H}^{\prime}} & -I_{k^{*}} & \frac{\partial^{2} \widetilde{l}_{M, t}}{\partial \omega_{M} \partial \widetilde{\theta}_{M}^{\prime}} \\
0 & 0 & 0 & \frac{\partial^{2} \widetilde{l}_{M, t}}{\partial \widetilde{\theta}_{M} \partial \widetilde{\theta}_{M}^{\prime}}
\end{array}\right],
\end{gathered}
$$

and in implementation we use a HAC estimator (e.g. Newey and West (1987)) to estimate $\mathcal{J}$.

Remark 6 With the covariance targeting specification in (9)-(10), the variation freeness property between the parameters of the HEAVY-P and HEAVY-V equations no longer holds, since $\kappa$ which appears in the HEAVY-P involves the parameter $\omega_{M}$ of the HEAVY-V equation. Thus, the block $\frac{\partial^{2} \widetilde{l}_{H, t}}{\partial \omega_{M} \partial \widetilde{\theta}_{H}^{\prime}}$ now appears in the Hessian to account for this dependence in the second step of estimation.

\section{Model Evaluation}

Our objective is to evaluate the out-of-sample performance of the HEAVY model compared to the GARCH model. We base our analysis on loss functions by defining an integrable loss function $L$ : $\mathbb{R}_{++}^{k \times k} \times \mathcal{H} \rightarrow \mathbb{R}_{+}$, where $\mathbb{R}_{++}^{k \times k}$ is the space of positive definite $(k \times k)$ matrices, and $\mathcal{H}$ is a compact subset of $\mathbb{R}_{++}^{k \times k}$ denoting the set of models under comparison. Let the loss function $L_{t, s}\left(\Sigma_{t+s}, H_{t+s \mid t}^{a}\right)$ denote the loss at time $t$ resulting from the $s$-step forecast using model $a$. The first argument of $L_{t, s}$ is the actual (unobserved) covariance matrix, $\Sigma_{t+s}$, and the second argument is its $s$-step forecast using model $a$ conditional on time $t$ information, $H_{t+s \mid t}^{a}$.

One practical issue is that $\Sigma_{t+s}$ is unobservable so the analysis will be based on some proxy denoted by $\widehat{\Sigma}_{t+s}$. In our empirical application, we use $V_{t+s}$ as proxy such that $\widehat{\Sigma}_{t+s}=V_{t+s}$. We use a quasi-likelihood (QLIK) loss function of the form

$$
L_{t, s}\left(\Sigma_{t+s}, H_{t+s \mid t}^{a}\right)=\log \left|H_{t+s \mid t}^{a}\right|+\operatorname{tr}\left(\left(H_{t+s \mid t}^{a}\right)^{-1} \widehat{\Sigma}_{t+s}\right),
$$


which provides a consistent ranking of volatility models in the sense of Patton (2011) and Patton and Sheppard (2009) as it is robust to noise in the proxy $\widehat{\Sigma}_{t+s}$; see also Laurent et al. (2009).

The loss function evaluates the $s$-step predicted density from model $a$ using the proxy $\widehat{\Sigma}_{t+s}$ as data. ${ }^{8}$ Note that even if - at time $t$ - the true density of $R_{t+1}$ is normal (i.e. the density of $P_{t+1}$ is Wishart), this result does not hold under temporal aggregation unless the conditional covariance matrix is constant. Therefore the $s$-step density will not be normal implying that the density used for the QLIK loss function (18) is misspecified. However, the loss difference between two competing models $a$ and $b, L_{t, s}\left(\Sigma_{t+s}, H_{t+s \mid t}^{a}\right)-L_{t, s}\left(\Sigma_{t+s}, H_{t+s \mid t}^{b}\right)$, can be interpreted as a KullbackLeibler distance which yields a valid assessment even if both models are misspecified.

Generally, if we let the true density of a random vector be denoted by $g(y)$ and the model density be denoted by $f(y ; \theta)$, then the Kullback-Leibler $(K L)$ distance is defined as

$$
K L=\int \log \left(\frac{g(y)}{f(y ; \theta)}\right) g(y) d y=\mathrm{E}_{g} \log g(y)-\mathrm{E}_{g} \log f(y ; \theta) .
$$

If the KL distance is used as a criterion for model comparison, the term involving the true density $g(y)$ drops out. For instance, a relative KL $(R K L)$ distance for two competing models $a$ and $b$ is given by

$$
\begin{aligned}
R K L(a, b) & =\left[\mathrm{E}_{g} \log g(y)-\mathrm{E}_{g} \log f^{a}\left(y ; \theta^{a}\right)\right]-\left[\mathrm{E}_{g} \log g(y)-\mathrm{E}_{g} \log f^{b}\left(y ; \theta^{b}\right)\right] \\
& =\mathrm{E}_{g} \log f^{b}\left(y ; \theta^{b}\right)-\mathrm{E}_{g} \log f^{a}\left(y ; \theta^{a}\right) .
\end{aligned}
$$

Although $\mathrm{E}_{g}(\cdot)$ is an expectation with respect to the true density, it can be consistently estimated using $T^{-1} \sum_{t=1}^{T} \log f_{t}\left(y_{t} ; \theta\right)$. Cox (1961) proposes a likelihood ratio test based on this approach. Vuong (1989) provides the theoretical framework in the case of nested and non-nested models. Similar approaches are proposed for out-of-sample model selection in Amisano and Giacomini (2007) and Diks et al. (2008).

We denote the loss difference between the HEAVY and GARCH models by

$$
D_{t, s}=L_{t, s}\left(\Sigma_{t+s}, H_{t+s \mid t}^{H E A V Y}\right)-L_{t, s}\left(\Sigma_{t+s}, H_{t+s \mid t}^{G A R C H}\right), \quad t=Q, Q+1, \ldots, T-s,
$$

where $L_{t, s}(\cdot)$ is given by (18), $T$ is the size of the full sample and $Q$ is the size of the estimation window. We assume $Q$ is fixed so that we use a rolling-window of data to estimate the model parameters, which gives $T-Q-s+1$ data points for out-of-sample model evaluation. The average loss is denoted by

$$
\bar{D}_{s}=\frac{1}{T-Q-s+1} \sum_{t=Q}^{T-s} D_{t, s}
$$

which is used to test $H_{0}: E\left[D_{t, s}\right]=0$, for all $s$, against a two-sided alternative. Let $\bar{D}_{s}^{*}$ denote the average loss evaluated at the true parameter value, then we have

\footnotetext{
${ }^{8}$ Note (18) is the negative of the log-likelihood of a multivariate normal density excluding the constant terms. The switched sign is due to defining (18) as a "loss" function.
} 


$$
\sqrt{T}\left(\bar{D}_{s}-\bar{D}_{s}^{*}\right) \stackrel{d}{\longrightarrow} N\left(0, \Lambda_{s}\right)
$$

where $\Lambda_{s}$ is the asymptotic variance of $D_{t, s}$ estimated using a HAC estimator (e.g. Newey and West (1987)). Significantly negative values of the test statistic indicate superior forecast performance of the HEAVY model. This predictive ability test was first introduced by Diebold and Mariano (1995), and later formalized by West (1996) and Giacomini and White (2006).

We extend this strategy in the context of multivariate volatility models by conducting separate tests for forecasts of the individual variances and also for the dependence structure of the group of assets under consideration. Consider the margins-copula decomposition of the log-likelihood of $R_{t}$,

$$
\log f(X)=\sum_{i=1}^{k} \log f_{i}\left(x_{i}\right)+\log c\left(F_{1}\left(x_{1}\right), F_{2}\left(x_{2}\right), \ldots, F_{k}\left(x_{k}\right)\right),
$$

where $f(X)$ is the joint density of the returns of the $k$ assets, $f_{i}\left(x_{i}\right)$ and $F_{i}\left(x_{i}\right), i=1, \ldots, k$, are respectively the density and cumulative distribution function of asset $i$ returns, and $c(\cdot)$ is the copula density. ${ }^{9}$ The normality assumption for $R_{t}$ implies that $f(X), f_{i}\left(x_{i}\right)$ and $c$ correspond to the multivariate normal density, normal density and normal copula, respectively.

We decompose the QLIK loss in (18) in a similar fashion to (19). So computing the loss in (18) based on the whole forecast matrix $\left(H_{t+s \mid t}^{a}\right)$ corresponds to $\log f(X)$, while computing the loss based on a particular diagonal element of $H_{t+s \mid t}^{a}$, say $h_{i i, t+s \mid t}^{a}$, corresponds to $\log f_{i}\left(x_{i}\right)$. The latter corresponds to the loss encountered in forecasting the individual variance for asset $i$, and we compute it for all $k$ assets. We compute the loss attributed to forecasting the dependence structure (summarized by the copula contribution) as the residual, i.e. corresponding to $\log f(X)-$ $\sum_{i=1}^{k} \log f_{i}\left(x_{i}\right)$. Based on this QLIK loss decomposition, we conduct the predictive ability test, outlined above, separately for each margin (i.e. individual variance) as well as the copula. Due to the normality assumption, the copula parameter is the conditional correlation matrix of the daily returns, thus we use the terms margins-copula and variances-correlations interchangeably.

\section{Empirical Application}

We use high-frequency data on Spyder (SPY), an S\&P 500 exchange traded fund, along with some of the most liquid stocks in the Dow Jones Industrial Average (DJIA) index. These are: Alcoa (AA), American Express (AXP), Bank of America (BAC), Coca Cola (KO), Du Pont (DD), General Electric (GE), International Business Machines (IBM), JP Morgan (JPM), Microsoft (MSFT), and Exxon Mobil (XOM). The sample period is $1 / 2 / 2001$ to $31 / 12 / 2009$ and the source of the data is the TAQ database. We choose the starting date for the sample to be after decimal pricing had been fully implemented in the NYSE, which took place on 29/1/2001.

\footnotetext{
${ }^{9}$ Nelsen (2006) and Patton (2009) provide recent reviews of copula theory and financial applications.
} 
We focus on the realized covariance matrix as our choice for $V_{t}$. In estimating the realized covariance matrix, we use 5-minute returns with subsampling. We exclude the opening and closing 15 minutes of trading to control for overnight effects. For the daily return, we focus on the opento-close returns which of course ignore overnight effects, and for consistency with the realized covariance estimator we compute the open-to-close daily returns over the same interval. Our estimation and forecast evaluation computations were repeated using the noise-robust realized kernel of Barndorff-Nielsen et al. (2011) with the results being qualitatively similar in general. ${ }^{10}$

The main focus of our empirical application will be on modelling and forecasting the conditional covariance matrix of a stock (BAC) and an index (S\&P 500) using the scalar HEAVY model. Most of the model's features can be readily seen in this bivariate model which is analyzed in 5.1.1. In 5.1.2, we analyze other pairs of assets using the scalar HEAVY model. In 5.2, we report estimation and forecast evaluation results for the bivariate diagonal HEAVY model and highlight differences from the scalar HEAVY case. Finally, in 5.3 we report estimates for the scalar HEAVY model with 10 assets using covariance targeting.

\subsection{Bivariate Scalar HEAVY Model}

\subsubsection{S\&P 500 and Bank of America}

Figure 1 contains the annualized realized volatility of SPY and BAC, their realized correlation and realized beta for BAC over the full sample. The sharp increase in volatility in 2008-2009 is associated with the turmoil in financial markets during the recent financial crisis. The increase in BAC volatility is much more pronounced especially after the collapse of Lehman Brothers in mid September 2008. BAC realized correlation with the market seems to have been relatively high during the crisis, and its realized beta increased sharply and was very volatile during this period.

For the scalar HEAVY model, the parameters $\bar{A}_{H}$ and $\bar{B}_{H}$ in (5) are replaced with $\bar{a}_{H} I_{k}$ and $\bar{b}_{H} I_{k}$, respectively, where $\bar{a}_{H}$ and $\bar{b}_{H}$ are scalars. The same applies to (6). The quadratic forms in (5) and (6) imply that the scalar HEAVY parameterization is

$$
H_{t}=\bar{C}_{H} \bar{C}_{H}^{\prime}+\bar{b}_{H}^{2} H_{t-1}+\bar{a}_{H}^{2} V_{t-1}, \quad M_{t}=\bar{C}_{M} \bar{C}_{M}^{\prime}+\bar{b}_{M}^{2} M_{t-1}+\bar{a}_{M}^{2} V_{t-1}
$$

In the vech representation (7), $A_{H}$ and $B_{H}$ are replaced with $\bar{a}_{H}^{2} I_{k^{*}}$ and $\bar{b}_{H}^{2} I_{k^{*}}$, respectively, and the same applies to the dynamic parameters in (8). So the vech parameterization is

$$
h_{t}=C_{H}+\bar{b}_{H}^{2} h_{t-1}+\bar{a}_{H}^{2} v_{t-1}, \quad m_{t}=C_{M}+\bar{b}_{M}^{2} m_{t-1}+\bar{a}_{M}^{2} v_{t-1},
$$

where $C_{H}=L_{k}\left(\bar{C}_{H} \otimes \bar{C}_{H}\right) D_{k} v e c h\left(I_{k}\right)$ and $C_{M}=L_{k}\left(\bar{C}_{M} \otimes \bar{C}_{M}\right) D_{k} v e c h\left(I_{k}\right)$. The parameterization of the corresponding GARCH and GARCH-X specifications is analogous, where in the GARCH$\mathrm{X}$ model we use $A_{G X}$ and $D_{G X}$ to denote the coefficients on $v_{t-1}$ and $p_{t-1}$, respectively. We include the estimates of the GARCH and GARCH-X models for comparison with the HEAVY-P

\footnotetext{
${ }^{10}$ These are not reported in the interest of parsimony, but are available upon request.
} 

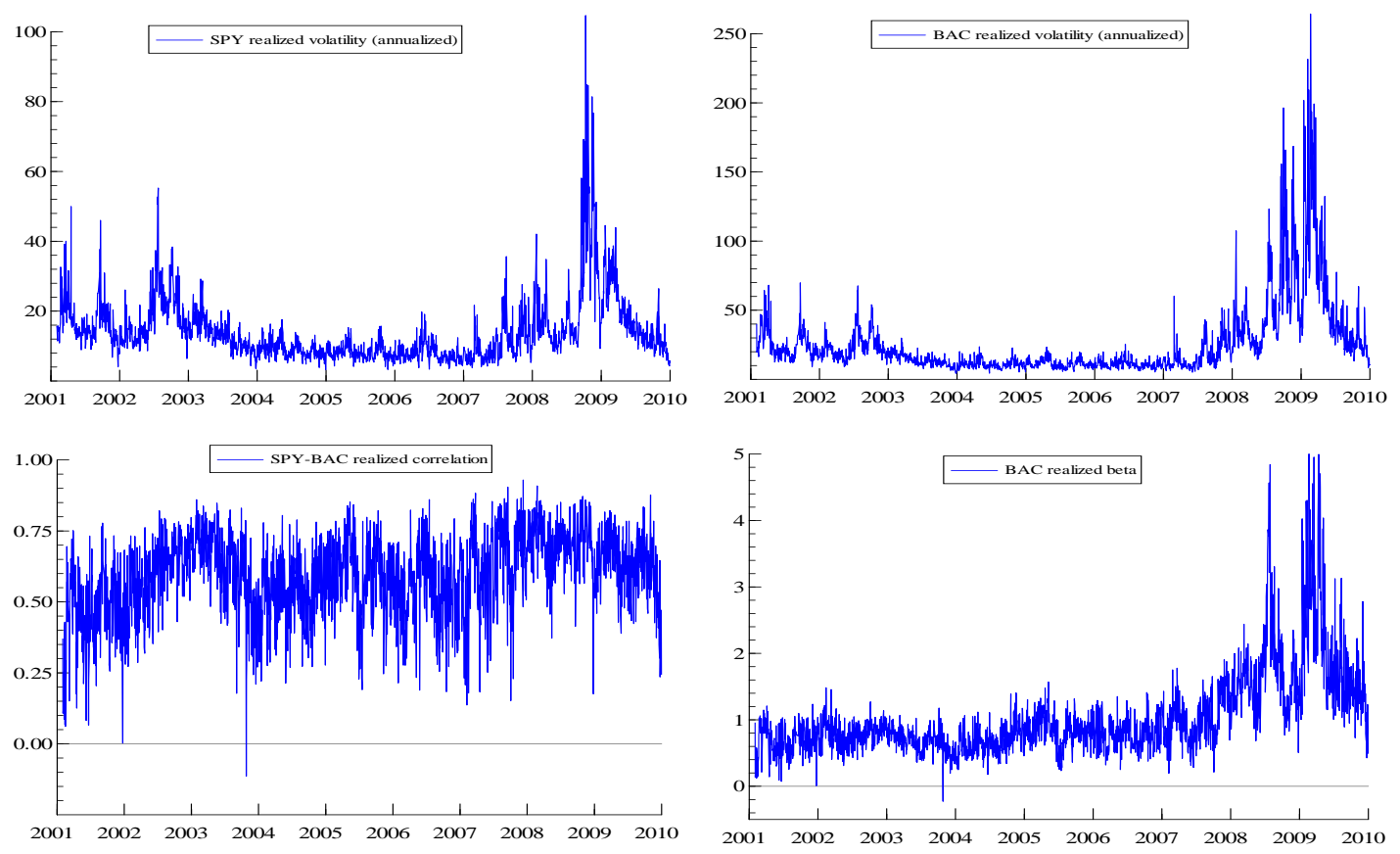

Figure 1: SPY and BAC annualized realized volatility, realized correlation and BAC realized beta.

equation. In Table 2, we present the parameter estimates, the log-likelihoods at the estimated parameters and the decomposition of the log-likelihood of the HEAVY-P and GARCH equations into their respective margins' and copula log-likelihoods. For ease of interpretation, we only report the parameter estimates for the models' vech representation excluding the constant terms unless otherwise stated.

The estimate of $B_{H}$ implies that the elements of $H_{t}$ will be smooth, although less smooth than the corresponding estimates from the GARCH model with the estimate of $B_{G}$ equal to 0.934 . For the HEAVY-V equation, the $B_{M}$ coefficient is relatively small implying that the estimated conditional moments will be somewhat erratic. In terms of magnitude, these estimates are largely in line with the estimates from the univariate HEAVY model in Shephard and Sheppard (2010), and they also suggest a somewhat high level of persistence. Compared to the nesting GARCH-X model, there is no loss of fit when moving to the HEAVY-P model since the coefficient on $p_{t-1}$ $\left(D_{G X}\right)$ is not statistically significant. This is not the case when moving from GARCH-X to GARCH which suggests that $v_{t-1}$ effectively crowds out $p_{t-1}$.

The log-likelihood and its decomposition into marginal and copula likelihoods indicate an improvement in fit of the HEAVY-P equation compared to the GARCH model. Note that the two models are non-nested so direct LR tests are not possible; however, we will present below the outcome of the predictive ability tests discussed in Section 4. Although non-nested, the decomposition suggests that the HEAVY-P equation improves on GARCH for both the margins and the copula. 


\begin{tabular}{ccccccccccc}
\hline & \multicolumn{2}{c}{ HEAVY-P } & \multicolumn{2}{c}{ GARCH } & \multicolumn{3}{c}{ GARCH-X } & \multicolumn{3}{c}{ HEAVY-V } \\
& $A_{H}$ & $B_{H}$ & $A_{G}$ & $B_{G}$ & $A_{G X}$ & $B_{G X}$ & $D_{G X}$ & $A_{M}$ & $B_{M}$ \\
& 0.214 & 0.727 & 0.062 & 0.934 & 0.187 & 0.741 & 0.019 & 0.421 & 0.574 \\
SPY-BAC & $\left(\begin{array}{c}\text { (st. error) } \\
\hline\end{array}\right.$ & $(0.054)$ & $(0.068)$ & $(0.010)$ & $(0.011)$ & $(0.056)$ & $(0.068)$ & $(0.012)$ & $(0.033)$ & $(0.033)$ \\
\hline
\end{tabular}

\begin{tabular}{lccc} 
& \multicolumn{2}{c}{ Log-likelihood decomposition $($ HEAVY-P versus GARCH) } \\
& HEAVY-P & GARCH & HEAVY-P gains \\
Margin 1 (SPY) & -658 & -713 & 55 \\
Margin 2 (BAC) & -1593 & -1648 & 55 \\
Copula & 815 & 808 & 7 \\
Joint distribution & -1436 & -1553 & 117 \\
\hline
\end{tabular}

Predictive ability tests at different forecast horizons (days)

\begin{tabular}{lcccccc} 
& $(1)$ & $(2)$ & $(3)$ & $(5)$ & $(10)$ & $(22)$ \\
Margin 1 (SPY) & -3.72 & -3.03 & -2.33 & -1.23 & 0.84 & 1.87 \\
Margin 2 (BAC) & -3.27 & -2.45 & -1.70 & -0.58 & 1.06 & 2.04 \\
Copula & -3.37 & -3.22 & -3.39 & -3.28 & -3.26 & -3.85 \\
Joint distribution & -4.32 & -3.78 & -3.23 & -2.33 & -0.07 & 1.03 \\
\hline
\end{tabular}

Table 2: Scalar HEAVY estimation and forecast evaluation results for SPY-BAC. Upper panel: parameter estimates of HEAVY, GARCH and GARCH-X with standard errors reported in parentheses. Middle panel: decomposition of the log-likelihood at the estimated parameter values. Bottom panel: t-statistics of the predictive ability tests for HEAVY versus GARCH.

The fit of the model can be analyzed through the residual plots shown in Figure 2. In $P_{t}=$ $H_{t}^{\frac{1}{2}} \varepsilon_{t} H_{t}^{\frac{1}{2}}$ and $V_{t}=M_{t}^{\frac{1}{2}} \eta_{t} M_{t}^{\frac{1}{2}}$, it is assumed that $\varepsilon_{t}$ and $\eta_{t}$ are Wishart distributed. This implies that for daily returns $R_{t}=H_{t}^{\frac{1}{2}} \xi_{t}, \xi_{t} \stackrel{i . i . d .}{\sim} N\left(0, \mathrm{I}_{k}\right)$, where $\varepsilon_{t}=\xi_{t} \xi_{t}^{\prime}$. Figure 2 plots the elements of $\widehat{\xi}_{t}=\widehat{H}_{t}^{-\frac{1}{2}} R_{t}$ in the left panel and $\widehat{\eta}_{t}=\widehat{M}_{t}^{-\frac{1}{2}} V_{t} \widehat{M}_{t}^{-\frac{1}{2}}$ in the right panel, where $\widehat{H}_{t}$ and $\widehat{M}_{t}$ are the estimated conditional moments, and $\widehat{\xi}_{t}$ and $\widehat{\eta}_{t}$ are the residuals from fitting the model. The elements of $\widehat{\xi}_{t}$ (assumed to be a random vector with a standard multivariate normal distribution) seem to have expectation zero as assumed, and their covariance hovers around zero. For $\widehat{\eta}_{t}$ (assumed to be a random matrix following a standardized Wishart distribution with an identity scale matrix), its diagonal elements seem to be centred around one, and the off-diagonal element $\left(\widehat{\eta}_{12, t}\right)$ is roughly centred around zero. ${ }^{11}$

Another interesting feature from the residual analysis is that it displays evidence of the leverage effect between the returns and the realized measure. This is shown in Figure 3. The upper-left chart shows the scatter plot of the $\widehat{\xi}_{1, t}=\widehat{H}_{11, t}^{-\frac{1}{2}} r_{1, t}$ and $\widehat{\eta}_{11, t}=\widehat{M}_{11, t}^{-\frac{1}{2}} V_{11, t} \widehat{M}_{11, t}^{-\frac{1}{2}}$ which are the innovations to the daily return and realized measure of SPY, respectively. ${ }^{12}$ The lower-left chart displays the innovations to the daily return and realized measure of BAC. The right panel charts correspond to the same plots but mapped into copula space where the empirical distribution function is used

\footnotetext{
${ }^{11}$ To improve the plots, we removed two large outliers in the HEAVY-V equation residuals corresponding to the realized variances of SPY and BAC on $27 / 2 / 2007$, due to the $9 \%$ fall in the Shanghai stock exchange index that day.

${ }^{12}$ We use $R_{1, t}$ to denote the first element of $R_{t}$, which is the SPY return on day $t$.
} 

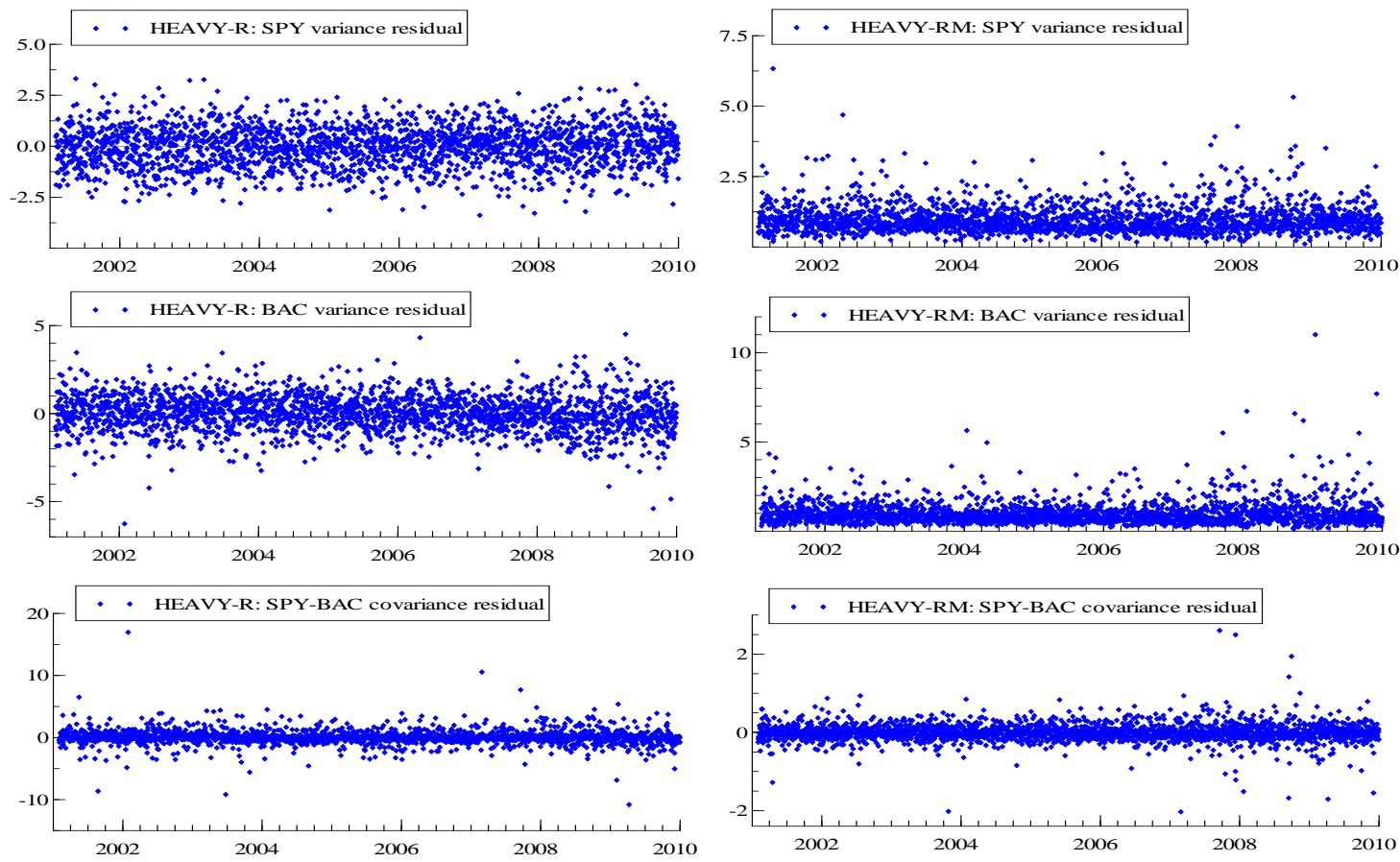

Figure 2: Residual plots for the SPY-BAC scalar HEAVY model.

to transform the innovations into probability integral transforms. The leverage effect can be seen clearly in the right panel. For instance, large negative innovations to SPY returns tend to be associated with large positive innovations to the realized measure indicating higher volatility in response to bad news. The same applies to BAC innovations.

The bottom panel of Table 2 gives the results of the predictive ability tests. We estimate the model using a rolling-window of 1486 observations and then use the parameter estimates to obtain forecasts of $H_{t}$ at horizons $s=1,2,3,5,10,22$ days using (14). The size of the rolling window is chosen such that our forecasts start at 3/1/2007. The reported figures are $t$-statistics to test equal predictive ability and significantly negative $t$-statistics favour the HEAVY model over the GARCH model. The results show that the HEAVY model forecasts outperform GARCH forecasts especially at short forecast horizons. This is true for the whole covariance matrix forecast as well its decomposition into its margins and copula, which provides further insight into the source of the forecast gains. The copula gains are maintained at longer forecast horizons indicating that the realized measure provides valuable information for forecasting the conditional correlation.

As pointed out earlier, the forecast profile of the HEAVY model is distinct from that of the GARCH model particularly over short forecast horizons due to momentum effects. This can be seen by considering the forecasts of the SPY-BAC conditional correlation (implied by the forecasts of $H_{t}$ ) over the period $03 / 11 / 2008$ to $30 / 09 / 2009$. This is an interesting period for analysis as it marks 

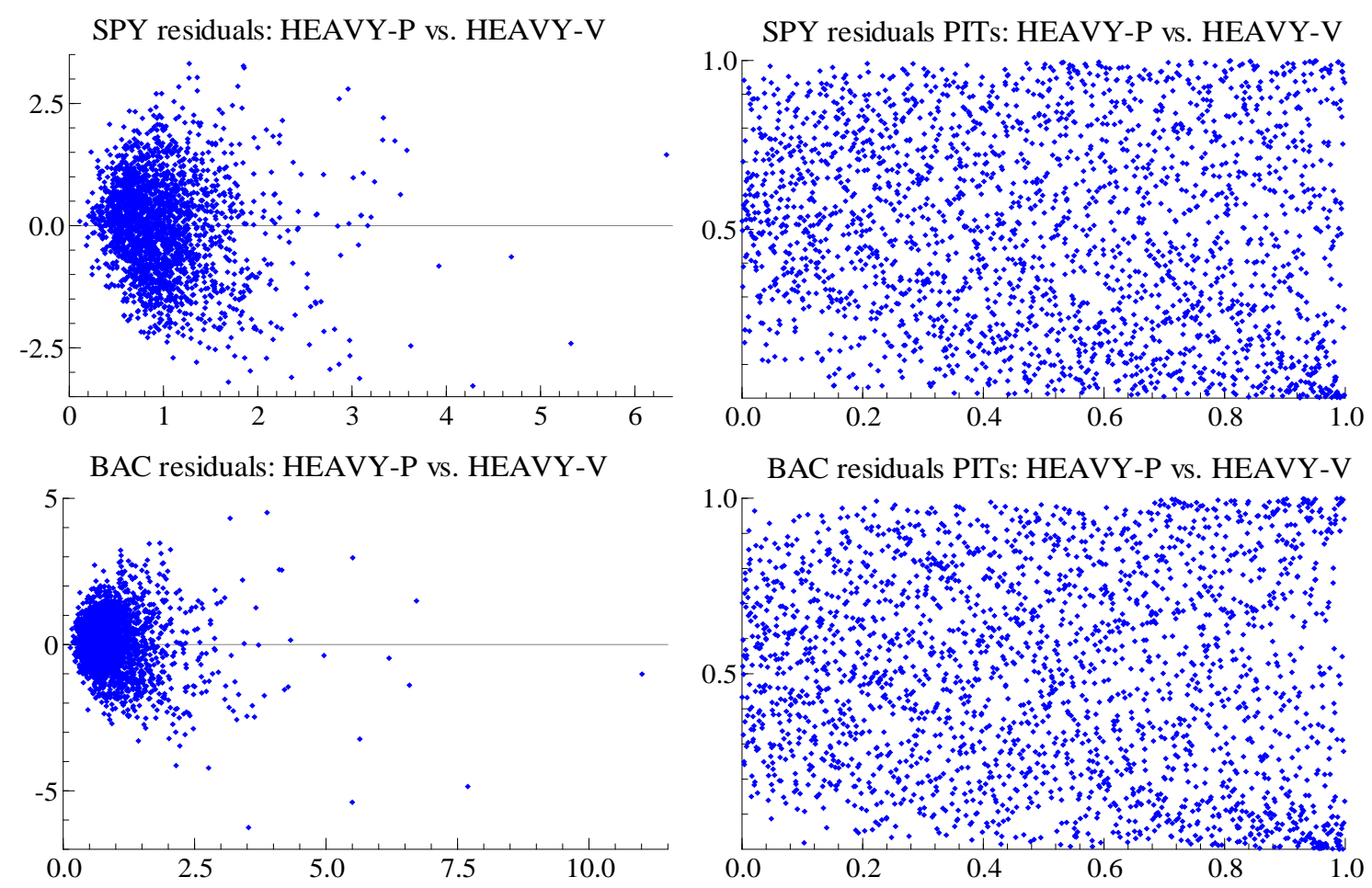

Figure 3: Left panel: scatter plots of SPY and BAC residuals in the HEAVY-P and HEAVY-V equations. Right panel: same plots mapped into probability integral transforms (PITs).

a very volatile period during the 2007-2009 financial crisis. The solid lines are the 1-step forecasts, and at selected points we plot - using dashed lines - the forecast profile at this date for 22 days into the future. We do this only for selected peak and trough points for clarity of illustration. The momentum effects in the HEAVY model can be readily seen. Whereas the GARCH correlation forecasts monotonically mean reverts, the HEAVY forecast displays some short run momentum influenced by the deviation of the realized measure from its long-run value before ultimately mean reverting. Interestingly, the plot also shows how the 1-step forecasts from both models diverge in some periods pointing to important differences in the information content of the realized measure and the outer product of daily returns.

It is interesting to track the model's performance in relation to the accuracy of the realized measure. For this purpose, we report in Table 3 the parameter estimates, log-likelihood gains and out-of-sample performance using various sampling intervals for the realized covariance estimator. The table also includes results when using the realized kernel as the realized measure. In general, the results indicate that when sampling between 5 and 15 minutes, the parameters estimates of the HEAVY and GARCH-X models are rather stable implying similar persistence levels, and indeed the estimates become very close when sampling at 30 minutes. At 1-minute sampling, there is substantial drop in the estimate of $B_{H}$ and a moderate increase in $A_{H}$. Using the realized kernel 


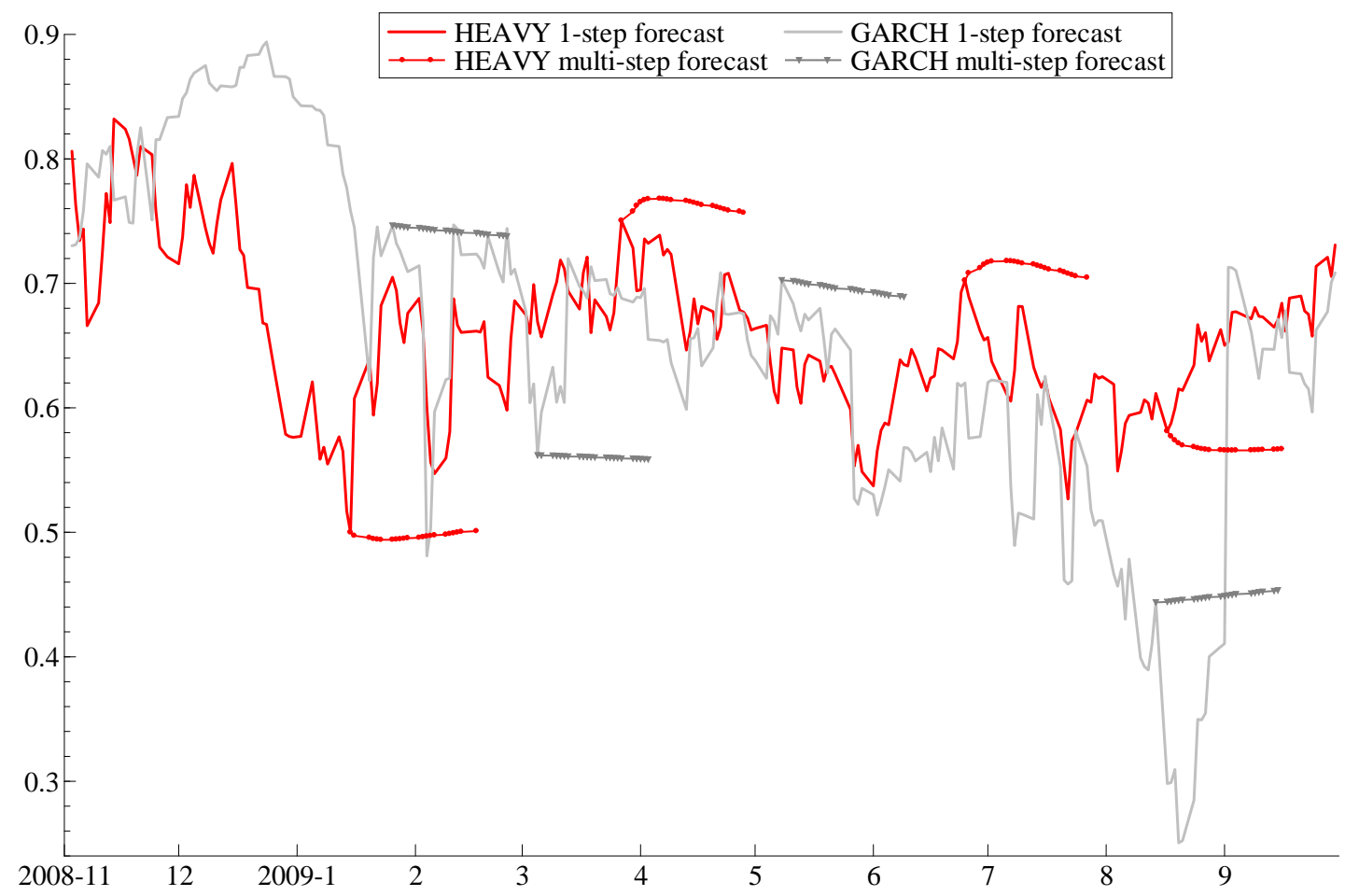

Figure 4: One-step and multi-step forecasts for the SPY-BAC conditional correlation.

leads to a noticeable decline in the smoothing parameters in both equations of the HEAVY model as well as the GARCH-X model. In terms of forecasting performance, the results are similar.

\subsubsection{Other Asset Pairs}

We also estimate the scalar HEAVY model for other pairs of assets in the DJIA index. The pairing of the assets is chosen by selecting companies in the same sector (e.g. BAC-JPM and IBM-MSFT), where we expect more persistent correlation dynamics, and also pairs of companies in different sectors. The objective is to track the HEAVY model's performance in each case. Table 4 reports the parameter estimates for the HEAVY, GARCH and GARCH-X models. One notable feature is that the estimates do not display large variation across the different pairs. As in the SPY-BAC case, inclusion of the realized measure crowds out the outer product of returns as the coefficient $D_{G X}$ is statistically insignificant in all cases.

The decomposition of the log-likelihood gains shows that the HEAVY model gains are obtained for each pair with respect to both margins and the copula with only one exception. The HEAVY model overall log-likelihood gain for the joint distribution is uniform across all pairs. The predictive ability test results indicate that the HEAVY model performs better than GARCH for all asset pairs, with the gains being particularly significant at short forecast horizons. We do not report 


\begin{tabular}{lcccccccc}
\hline & \multicolumn{2}{c}{ HEAVY-P } & \multicolumn{3}{c}{ GARCH-X } & \multicolumn{2}{c}{ HEAVY-V } & Joint LL \\
& $A_{H}$ & $B_{H}$ & $A_{G}$ & $B_{G}$ & $D_{G X}$ & $A_{M}$ & $B_{M}$ & gain/loss \\
RC 1-min & 0.256 & 0.597 & 0.128 & 0.741 & 0.052 & 0.527 & 0.471 & 65 \\
RC 5-min & 0.214 & 0.727 & 0.187 & 0.741 & 0.019 & 0.421 & 0.574 & 117 \\
RC 10-min & 0.202 & 0.760 & 0.189 & 0.764 & 0.011 & 0.362 & 0.633 & 120 \\
RC 15-min & 0.185 & 0.787 & 0.169 & 0.793 & 0.012 & 0.300 & 0.696 & 116 \\
RC 30-min & 0.143 & 0.842 & 0.134 & 0.843 & 0.009 & 0.236 & 0.759 & 107 \\
Realized kernel & 0.213 & 0.677 & 0.194 & 0.689 & 0.015 & 0.508 & 0.488 & 128 \\
\hline
\end{tabular}

Joint distribution predictive ability tests at different forecast horizons (days)

\begin{tabular}{lcccccc} 
& $(1)$ & $(2)$ & $(3)$ & $(5)$ & $(10)$ & $(22)$ \\
RC 1-min & -3.59 & -3.08 & -2.47 & -1.82 & 0.30 & 1.50 \\
RC 5-min & -4.32 & -3.78 & -3.23 & -2.33 & -0.08 & 1.03 \\
RC 10-min & -4.08 & -3.70 & -3.21 & -2.35 & -0.23 & 0.99 \\
RC 15-min & -4.26 & -3.87 & -3.34 & -2.64 & -0.26 & 1.11 \\
RC 30-min & -3.81 & -3.34 & -2.75 & -2.01 & -0.13 & 1.12 \\
Realized kernel & -4.25 & -3.76 & -3.23 & -2.63 & -0.46 & 1.00 \\
\hline
\end{tabular}

Table 3: Scalar HEAVY estimation and forecast evaluation results for SPY-BAC using different realized measures. Upper panel: scalar HEAVY and GARCH-X parameter estimates using different sampling intervals in constructing the realized covariance and also using the realized kernel. Log-likelihood gains are reported in last column. Bottom panel: t-statistics of the predictive ability tests for HEAVY versus GARCH.

the margins-copula decomposition for these tests in the interest of brevity, but they show that the HEAVY model gains are maintained for some of the margins and also for the copula of some of the pairs. In no case was the GARCH model significantly favoured at any horizon except for the BAC and JPM margins towards the end of the forecast horizon.

\subsection{Bivariate Diagonal HEAVY Model}

In this subsection we discuss the estimation and forecast evaluation results for the diagonal HEAVY model. We exclude the GARCH-X model results to improve presentation noting that its results are in line with the those of the scalar model. For the diagonal HEAVY model in the bivariate case, the parameters $\bar{A}_{H}$ and $\bar{B}_{H}$ are given by the diagonal matrices

$$
\bar{A}_{H}=\left(\begin{array}{cc}
\bar{a}_{11, H} & 0 \\
0 & \bar{a}_{22, H}
\end{array}\right), \quad \bar{B}_{H}=\left(\begin{array}{cc}
\bar{b}_{11, H} & 0 \\
0 & \bar{b}_{22, H}
\end{array}\right),
$$

and the same applies to $\bar{A}_{M}$ and $\bar{B}_{M}$. The top panel of Table 5 presents estimates of the diagonal elements of the parameter matrices in (5) and (6) in order along with those of the corresponding GARCH model. These are easier to interpret when expressed in terms of the parameters of the vech representation in (7) and (8), which are reported underneath. Note that if $\bar{A}_{H}$ is, for instance, a $(2 \times 2)$ diagonal matrix, then $A_{H}$ will be a $(3 \times 3)$ diagonal matrix; see Example 2 . The first and third diagonal elements of $A_{H}$ will be the squares of the diagonal elements of $\bar{A}_{H}$, and the second 


\begin{tabular}{lcccccccccc}
\hline & \multicolumn{1}{c}{ HEAVY-P } & \multicolumn{2}{c}{ GARCH } & \multicolumn{3}{c}{ GARCH-X } & \multicolumn{2}{c}{ HEAVY-V } \\
& $A_{H}$ & $B_{H}$ & $A_{G}$ & $B_{G}$ & $A_{G X}$ & $B_{G X}$ & $D_{G X}$ & $A_{M}$ & $B_{M}$ \\
BAC - JPM & 0.260 & 0.639 & 0.062 & 0.938 & 0.180 & 0.702 & 0.046 & 0.450 & 0.550 \\
IBM - MSFT & 0.179 & 0.762 & 0.051 & 0.941 & 0.135 & 0.794 & 0.026 & 0.309 & 0.676 \\
XOM - AA & 0.188 & 0.737 & 0.057 & 0.935 & 0.114 & 0.804 & 0.034 & 0.315 & 0.667 \\
AXP - DD & 0.201 & 0.743 & 0.045 & 0.951 & 0.183 & 0.754 & 0.010 & 0.357 & 0.638 \\
GE - KO & 0.220 & 0.727 & 0.039 & 0.957 & 0.205 & 0.740 & 0.007 & 0.344 & 0.651 \\
\hline
\end{tabular}

HEAVY-P log-likelihood gains/losses (+/-) relative to GARCH

\begin{tabular}{lcccc} 
& Margin 1 & Margin 2 & Copula & Joint LL \\
BAC - JPM & 44 & 58 & 8 & 110 \\
IBM - MSFT & 30 & 37 & 17 & 84 \\
XOM - AA & 44 & 31 & -7 & 69 \\
AXP - DD & 56 & 49 & 16 & 121 \\
GE - KO & 47 & 34 & 9 & 90 \\
\hline
\end{tabular}

Joint distribution predictive ability tests at different forecast horizons (days)

\begin{tabular}{lcccccc}
\multicolumn{5}{c}{ Joint distribution predictive ability tests at different forecast horizons (days) } \\
& $(1)$ & $(2)$ & $(3)$ & $(5)$ & $(10)$ & $(22)$ \\
PM & -4.09 & -3.41 & -2.78 & -1.98 & 0.45 & 1.32 \\
MSFT & -2.92 & -2.60 & -2.38 & -1.94 & -1.56 & -0.46 \\
AA & -2.76 & -2.09 & -2.05 & -1.76 & -1.32 & -0.80 \\
DD & -3.46 & -3.09 & -2.84 & -2.39 & -1.28 & 0.18 \\
& -2.80 & -2.66 & -2.41 & -2.21 & -0.91 & 1.00
\end{tabular}

Table 4: Scalar HEAVY estimation and forecast evaluation results for other pairs of assets. Upper panel: parameter estimates of HEAVY, GARCH and GARCH-X. Middle panel: HEAVY-P log-likelihood gains/losses relative to GARCH. Bottom panel: t-statistics of the predictive ability tests for HEAVY versus GARCH. 


\begin{tabular}{|c|c|c|c|c|c|c|c|c|c|c|c|c|}
\hline \multirow{4}{*}{$\underset{\text { (st. error) }}{\text { SPY-BAC }}$} & \multicolumn{4}{|c|}{ HEAVY-P } & \multicolumn{4}{|c|}{ GARCH } & \multicolumn{4}{|c|}{ HEAVY-V } \\
\hline & \multicolumn{2}{|c|}{$\bar{A}_{H}$} & \multicolumn{2}{|c|}{$\bar{B}_{H}$} & \multicolumn{2}{|c|}{$\bar{A}_{G}$} & \multicolumn{2}{|c|}{$\bar{B}_{G}$} & \multicolumn{2}{|c|}{$\bar{A}_{M}$} & \multicolumn{2}{|c|}{$\bar{B}_{M}$} \\
\hline & $\begin{array}{l}0.447 \\
(0.048)\end{array}$ & $\begin{array}{l}0.477 \\
(0.057)\end{array}$ & $\begin{array}{l}0.858 \\
(0.033)\end{array}$ & $\begin{array}{l}0.844 \\
(0.041)\end{array}$ & $\begin{array}{l}0.238 \\
(0.023)\end{array}$ & $\begin{array}{l}0.262 \\
(0.035)\end{array}$ & $\begin{array}{l}0.968 \\
(0.006)\end{array}$ & $\begin{array}{l}0.964 \\
(0.010)\end{array}$ & $\begin{array}{l}0.632 \\
(0.025)\end{array}$ & $\begin{array}{l}0.663 \\
(0.033)\end{array}$ & $\begin{array}{l}0.768 \\
(0.022)\end{array}$ & $\begin{array}{l}0.748 \\
(0.028)\end{array}$ \\
\hline & \multicolumn{2}{|c|}{$A_{H}$} & \multicolumn{2}{|c|}{$B_{H}$} & \multicolumn{2}{|c|}{$A_{G}$} & \multicolumn{2}{|c|}{$B_{G}$} & \multicolumn{2}{|c|}{$A_{M}$} & \multicolumn{2}{|c|}{$B_{M}$} \\
\hline Var. eqn. (SPY) & \multicolumn{2}{|c|}{0.200} & \multicolumn{2}{|c|}{0.736} & \multicolumn{2}{|c|}{0.057} & \multicolumn{2}{|c|}{0.938} & \multicolumn{2}{|c|}{0.400} & \multicolumn{2}{|c|}{0.590} \\
\hline Var. eqn. (BAC) & \multicolumn{2}{|c|}{0.228} & \multicolumn{2}{|c|}{0.713} & \multicolumn{2}{|c|}{0.069} & \multicolumn{2}{|c|}{0.929} & \multicolumn{2}{|c|}{0.439} & \multicolumn{2}{|c|}{0.560} \\
\hline Cov. eqn. & \multicolumn{2}{|c|}{0.213} & \multicolumn{2}{|c|}{0.725} & \multicolumn{2}{|c|}{0.063} & \multicolumn{2}{|c|}{0.933} & & & \multicolumn{2}{|c|}{0.575} \\
\hline
\end{tabular}

\begin{tabular}{|c|c|c|c|c|c|c|}
\hline \multicolumn{7}{|c|}{ Log-likelihood decomposition (HEAVY-P versus GARCH) } \\
\hline & \multicolumn{2}{|c|}{ HEAVY-P } & \multicolumn{2}{|c|}{ GARCH } & \multicolumn{2}{|c|}{ HEAVY-P gains } \\
\hline Margin 1 (SPY) & \multicolumn{2}{|c|}{-659} & \multicolumn{2}{|c|}{-713} & \multicolumn{2}{|c|}{54} \\
\hline Margin 2 (BAC) & \multicolumn{2}{|c|}{-1592} & \multicolumn{2}{|c|}{-1647} & \multicolumn{2}{|c|}{55} \\
\hline Copula & \multicolumn{2}{|c|}{816} & \multicolumn{2}{|c|}{809} & \multicolumn{2}{|c|}{7} \\
\hline Joint distribution & \multicolumn{2}{|c|}{-1435} & \multicolumn{2}{|c|}{-1552} & \multicolumn{2}{|c|}{117} \\
\hline \multicolumn{7}{|c|}{ Predictive ability tests at different forecast horizons (days) } \\
\hline & (1) & (2) & (3) & (5) & (10) & $(22)$ \\
\hline Margin 1 (SPY) & -3.86 & -3.24 & -2.77 & -1.78 & 0.27 & 1.50 \\
\hline Margin 2 (BAC) & -3.17 & -2.45 & -1.79 & -0.80 & 0.89 & 1.90 \\
\hline Copula & -3.29 & -3.02 & -3.04 & -3.04 & -2.99 & -3.88 \\
\hline Joint distribution & -4.38 & -3.79 & -3.27 & -2.54 & -0.46 & 0.70 \\
\hline
\end{tabular}

Table 5: Diagonal HEAVY estimation and forecast evaluation results for SPY-BAC. Upper panel: parameter estimates of HEAVY and GARCH with standard errors reported in parentheses. Middle panel: decomposition of the log-likelihood at the estimated parameter values. Bottom panel: t-statistics of the predictive ability tests for HEAVY versus GARCH.

diagonal element of $A_{H}$ will be the product of the two diagonal elements of $\bar{A}_{H}$.

The estimates of the diagonal elements are rather similar within each parameter matrix, except for the HEAVY-V equation. Since the diagonal HEAVY models nests the scalar HEAVY model, we can test for the restriction using a Wald test. The scalar restriction is not rejected for both the HEAVY and GARCH models at the 5\% significance level. The log-likelihood decomposition results are similar to the scalar model and show improvements in both margins and the copula. The bottom panel of Table 5 shows that the diagonal HEAVY model provides superior forecasts with the gains being particularly significant at short forecast horizons.

We also report estimation results for the diagonal model using other pairs of assets in Table 6. For brevity, we only report parameter estimates for the vech representation. The parameter estimates show some variation within and across pairs. The Wald test results indicate that the scalar model restrictions are rejected at the $5 \%$ significance level only for the XOM-AA pair in the HEAVY-P equation, and only for the AXP-DD pair in the GARCH model. The scalar model restrictions for the HEAVY-V equation are not rejected in any of the pairs. The figures in the middle panel shows that the HEAVY model gains over GARCH in terms of the joint distribution log-likelihood are uniform across all pairs. The gains in the margins and the copula - not reported 


\begin{tabular}{lcccccc}
\hline & \multicolumn{2}{c}{ HEAVY-P } & \multicolumn{2}{c}{ GARCH } & \multicolumn{2}{c}{ HEAVY-V } \\
& $A_{H}$ & $B_{H}$ & $A_{G}$ & $B_{G}$ & $A_{M}$ & $B_{M}$ \\
Variance (BAC) & 0.267 & 0.638 & 0.051 & 0.947 & 0.433 & 0.566 \\
Variance (JPM) & 0.256 & 0.634 & 0.074 & 0.925 & 0.473 & 0.526 \\
Covariance (BAC-JPM) & 0.262 & 0.636 & 0.061 & 0.936 & 0.452 & 0.546 \\
Variance (IBM) & 0.187 & 0.761 & 0.052 & 0.939 & 0.331 & 0.652 \\
Variance (MSFT) & 0.172 & 0.764 & 0.049 & 0.945 & 0.291 & 0.695 \\
Covariance (IBM-MSFT) & 0.180 & 0.763 & 0.050 & 0.942 & 0.311 & 0.673 \\
Variance (XOM) & 0.175 & 0.713 & 0.073 & 0.907 & 0.338 & 0.644 \\
Variance (AA) & 0.180 & 0.784 & 0.043 & 0.952 & 0.285 & 0.698 \\
Covariance (XOM-AA) & 0.178 & 0.748 & 0.056 & 0.929 & 0.310 & 0.670 \\
Variance (AXP) & 0.218 & 0.738 & 0.065 & 0.931 & 0.371 & 0.628 \\
Variance (DD) & 0.186 & 0.740 & 0.031 & 0.963 & 0.338 & 0.645 \\
Covariance (AXP-DD) & 0.201 & 0.739 & 0.045 & 0.947 & 0.354 & 0.636 \\
Variance (GE) & 0.211 & 0.750 & 0.042 & 0.956 & 0.354 & 0.645 \\
Variance (KO) & 0.283 & 0.610 & 0.037 & 0.957 & 0.331 & 0.653 \\
Covariance (GE-KO) & 0.245 & 0.676 & 0.039 & 0.956 & 0.342 & 0.649 \\
\hline
\end{tabular}

Log-likelihood decomposition (HEAVY-P versus GARCH)

\begin{tabular}{lccc} 
& HEAVY-P & GARCH & HEAVY-P gains \\
BAC - JPM & -2828 & -2936 & 108 \\
IBM - MSFT & -2295 & -2380 & 85 \\
XOM - AA & -3415 & -3485 & 71 \\
AXP - DD & -3155 & -3271 & 116 \\
GE - KO & -2211 & -2304 & 93 \\
\hline
\end{tabular}

Predictive ability tests at different forecast horizons (days)

\begin{tabular}{lcccccc} 
& $(1)$ & $(2)$ & $(3)$ & $(5)$ & $(10)$ & $(22)$ \\
BAC - JPM & -3.78 & -3.13 & -2.47 & -1.68 & 0.69 & 1.84 \\
IBM - MSFT & -2.91 & -2.59 & -2.39 & -1.94 & -1.57 & -0.49 \\
XOM - AA & -2.84 & -2.15 & -2.14 & -1.87 & -1.42 & -0.93 \\
AXP - DD & -3.24 & -2.94 & -2.75 & -2.33 & -1.43 & -0.24 \\
GE - KO & -2.87 & -2.71 & -2.50 & -2.25 & -1.04 & 0.83 \\
\hline
\end{tabular}

Table 6: Diagonal HEAVY parameter estimates for other pairs of assets. Upper panel: parameter estimates of HEAVY and GARCH. All coefficients are statistically significant at the 5 percent significance level. Middle panel: HEAVY-P and GARCH log-likelihood at the estimated parameter values. Bottom panel: t-statistics of the predictive ability tests for HEAVY versus GARCH. 
for brevity - mirror the results in the scalar model; see middle panel of Table 4. The t-statistics of the predictive ability tests in the bottom panel indicate that the HEAVY model consistently outperforms the GARCH model.

\subsection{Covariance Targeting Scalar HEAVY Model}

In this section, we estimate the scalar HEAVY model including all 10 DJIA assets. We show the estimation results for both the original HEAVY specification and the covariance targeting model given by (11)-(10). We focus on this covariance targeting specification since it is easier to handle the parameter restrictions required for covariance stationarity and positive definiteness of the target; see Remark 3. For the GARCH model, we also estimate its covariance targeting parameterization which has a similar structure to (10). With covariance targeting, the number of parameters to be estimated through numerical optimization is reduced from 57 to 2 parameters per equation, where the latter are the dynamic parameters of interest.

Table 7 presents the estimates of the dynamic parameters for the HEAVY and GARCH models. The parameter estimates show some differences compared to the average estimate from the bivariate models for the same assets. The estimates of the smoothing parameters $\left(B_{H}, B_{M}\right.$ and $\left.B_{G}\right)$ have all increased especially $B_{M}$, while the estimates of $\left(A_{H}, A_{M}\right.$ and $\left.A_{G}\right)$ are now smaller. The loglikelihood decomposition results show uniform gains for the HEAVY model in all margins and the copula. The copula gains seem particularly impressive. In terms of parameter estimates and the log-likelihood decomposition, the covariance targeting model shows only slight differences compared to the non-targeting specification.

In Figure 5, we present summary results of the predictive ability tests for the covariance targeting scalar HEAVY and GARCH models. The figure shows the t-statistics for tests for the joint distribution, copula and the minimum, maximum, and median t-statistics for the ten margins. In the first three days, the HEAVY model gains are confirmed for the joint distribution, all margins and the copula. The gains of the joint distribution are maintained up to 11 days ahead, then it falls into the insignificance region before improving again towards the end of the forecast horizon. For the margins, the median t-statistics show gains up to 7 days ahead. The copula gains are maintained throughout until the end of the forecast horizon, which is consistent with the substantial overall gain in the copula log-likelihood discussed above.

\section{Conclusion}

This paper introduces a new class of multivariate volatility models with robust performance in outof-sample prediction of the covariance matrix for a collection of financial assets. While GARCH models - in their many variations - have proved successful in the past two decades, the increasing availability of high-frequency data provides important additional information. Utilizing this infor-

mation to forecast the conditional variance of daily asset returns has already borne fruit in the 


\begin{tabular}{|c|c|c|c|c|c|c|}
\hline \multicolumn{7}{|c|}{ Scalar HEAVY model } \\
\hline & \multicolumn{2}{|c|}{ HEAVY-P } & \multicolumn{2}{|c|}{ GARCH } & \multicolumn{2}{|c|}{ HEAVY-V } \\
\hline \multirow[b]{2}{*}{$\underset{\substack{\text { Dynamic param. } \\
\text { (st. error) }}}{ }$} & $A_{H}$ & $B_{H}$ & $A_{G}$ & $B_{G}$ & $A_{M}$ & $B_{M}$ \\
\hline & $\begin{array}{l}0.141 \\
(0.021) \\
\end{array}$ & $\begin{array}{l}0.792 \\
(0.037) \\
\end{array}$ & $\begin{array}{l}0.024 \\
(0.002) \\
\end{array}$ & $\begin{array}{l}0.973 \\
(0.001) \\
\end{array}$ & $\begin{array}{l}0.247 \\
(0.011) \\
\end{array}$ & $\begin{array}{l}0.744 \\
(0.010) \\
\end{array}$ \\
\hline \multicolumn{7}{|c|}{ Log-likelihood decomposition } \\
\hline & \multicolumn{2}{|c|}{ HEAVY-P } & \multicolumn{2}{|c|}{ GARCH } & \multicolumn{2}{|c|}{ HEAVY-P gains } \\
\hline Margin 1 (BAC) & \multicolumn{2}{|c|}{-1611} & \multicolumn{2}{|c|}{-1696} & \multicolumn{2}{|c|}{85} \\
\hline Margin $2(\mathrm{JPM})$ & \multicolumn{2}{|c|}{-1999} & \multicolumn{2}{|c|}{-2098} & \multicolumn{2}{|c|}{99} \\
\hline Margin 3 (IBM) & \multicolumn{2}{|c|}{-1267} & \multicolumn{2}{|c|}{-1323} & \multicolumn{2}{|c|}{56} \\
\hline Margin 4 (MSFT) & \multicolumn{2}{|c|}{-1471} & \multicolumn{2}{|c|}{-1525} & \multicolumn{2}{|c|}{54} \\
\hline Margin 5 (XOM) & \multicolumn{2}{|c|}{-1331} & \multicolumn{2}{|c|}{-1420} & \multicolumn{2}{|c|}{89} \\
\hline Margin $6(\mathrm{AA})$ & \multicolumn{2}{|c|}{-2332} & \multicolumn{2}{|c|}{-2381} & \multicolumn{2}{|c|}{49} \\
\hline Margin 7 (AXP) & \multicolumn{2}{|c|}{-1957} & \multicolumn{2}{|c|}{-2034} & \multicolumn{2}{|c|}{77} \\
\hline Margin 8 (DD) & \multicolumn{2}{|c|}{-1530} & \multicolumn{2}{|c|}{-1595} & \multicolumn{2}{|c|}{65} \\
\hline Margin $9(\mathrm{GE})$ & \multicolumn{2}{|c|}{-1532} & \multicolumn{2}{|c|}{-1590} & \multicolumn{2}{|c|}{58} \\
\hline Margin $10(\mathrm{KO})$ & \multicolumn{2}{|c|}{-911} & & & & \\
\hline Copula & & & & & & \\
\hline Joint distribution & & & -1 & & & \\
\hline
\end{tabular}

\begin{tabular}{|c|c|c|c|c|c|c|}
\hline \multicolumn{7}{|c|}{ Covariance targeting scalar HEAVY model } \\
\hline & \multicolumn{2}{|c|}{ HEAVY-P } & \multicolumn{2}{|c|}{ GARCH } & \multicolumn{2}{|c|}{ HEAVY-V } \\
\hline \multirow[b]{2}{*}{$\begin{array}{c}\text { Dynamic param. } \\
\text { (st. error) }\end{array}$} & $A_{H}$ & $B_{H}$ & $A_{G}$ & $B_{G}$ & $A_{M}$ & $B_{M}$ \\
\hline & $\begin{array}{l}0.177 \\
(0.022) \\
\end{array}$ & $\begin{array}{l}0.818 \\
(0.023) \\
\end{array}$ & $\begin{array}{l}0.022 \\
(0.001)\end{array}$ & $\begin{array}{l}0.977 \\
(0.001)\end{array}$ & $\begin{array}{l}0.234 \\
(0.009)\end{array}$ & $\begin{array}{l}0.761 \\
(0.010)\end{array}$ \\
\hline \multicolumn{7}{|c|}{ Log-likelihood decomposition } \\
\hline & \multicolumn{2}{|c|}{ HEAVY-P } & \multicolumn{2}{|c|}{ GARCH } & \multicolumn{2}{|c|}{ HEAVY-P gains } \\
\hline Margin 1 (BAC) & \multicolumn{2}{|c|}{-1616} & \multicolumn{2}{|c|}{-1753} & \multicolumn{2}{|c|}{138} \\
\hline Margin 2 (JPM) & \multicolumn{2}{|c|}{-1985} & \multicolumn{2}{|c|}{-2119} & \multicolumn{2}{|c|}{133} \\
\hline Margin 3 (IBM) & \multicolumn{2}{|c|}{-1257} & \multicolumn{2}{|c|}{-1327} & \multicolumn{2}{|c|}{69} \\
\hline Margin 4 (MSFT) & \multicolumn{2}{|c|}{-1464} & \multicolumn{2}{|c|}{-1525} & \multicolumn{2}{|c|}{61} \\
\hline Margin $5(\mathrm{XOM})$ & \multicolumn{2}{|c|}{-1340} & \multicolumn{2}{|c|}{-1424} & \multicolumn{2}{|c|}{84} \\
\hline Margin $6(\mathrm{AA})$ & \multicolumn{2}{|c|}{-2324} & \multicolumn{2}{|c|}{-2379} & \multicolumn{2}{|c|}{55} \\
\hline Margin 7 (AXP) & \multicolumn{2}{|c|}{-1940} & \multicolumn{2}{|c|}{-2046} & \multicolumn{2}{|c|}{106} \\
\hline Margin 8 (DD) & \multicolumn{2}{|c|}{-1528} & \multicolumn{2}{|c|}{-1592} & \multicolumn{2}{|c|}{64} \\
\hline Margin $9(\mathrm{GE})$ & \multicolumn{2}{|c|}{-1521} & \multicolumn{2}{|c|}{-1595} & \multicolumn{2}{|c|}{74} \\
\hline Margin $10(\mathrm{KO})$ & \multicolumn{2}{|c|}{-911} & & & & \\
\hline Copula & & & & & & \\
\hline Joint distribution & & & -1 & 84 & & \\
\hline
\end{tabular}

Table 7: Scalar HEAVY estimates for 10 DJIA assets. Upper panel: parameter estimates and log-likelihood decomposition for scalar HEAVY and GARCH without covariance targeting. Bottom panel: parameter estimates and log-likelihood decomposition for the covariance targeting scalar HEAVY and GARCH. 


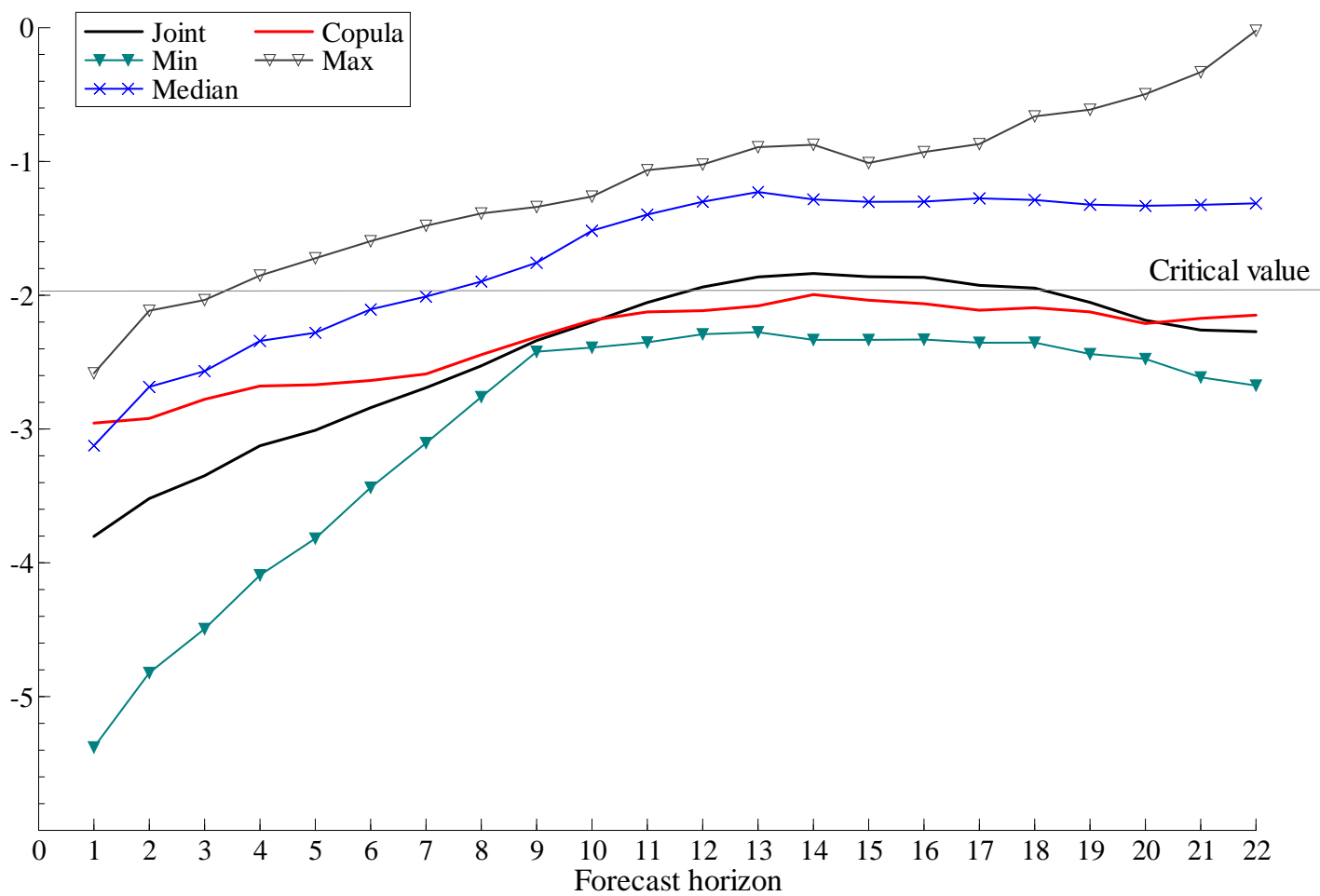

Figure 5: Predictive ability tests' t-statistics for the covariance targeting scalar HEAVY and GARCH models.

univariate case as documented by several recent studies.

Our study is one of the first to document this feature in the multivariate case using a relatively large group of assets. We present our results in the framework of the multivariate HEAVY class of models. Using a linear specification, we discuss in some detail the model's dynamic properties, its covariance targeting representation, and provide closed-form forecasting formulas. We show how the profile of forecasts from HEAVY models differs from GARCH models, in particular with regard to its persistence and short-run momentum effects. We also discuss QMLE of HEAVY models under the assumption of a Wishart distribution for the innovation matrices.

In an application to an S\&P 500 ETF and 10 stocks from the DJIA index, we put the HEAVY and GARCH models to the challenging test of forecasting the assets' conditional covariance matrix during the recent financial crisis. We show that forecasts from the HEAVY model dominate GARCH forecasts with the gains being particularly significant at short forecast horizons. The results seem consistent across different pairs of assets and also when using all ten assets in a covariance targeting model. The HEAVY model's relatively short memory compared to GARCH models seems to enable it to efficiently track sudden changes in volatility and the dependence structure of the assets. With 
regard to the latter, our results for overall log-likelihood decompositions and predictive ability tests strongly suggest that high-frequency data provides quite important information for modelling and forecasting conditional correlations.

For future research, a number of extensions could potentially add to our understanding of how best to model and forecast multivariate volatility. It would be interesting to add asymmetric effects to the HEAVY model to explicitly capture the leverage effect and see how this improves its forecast

performance. It might also be beneficial to use a long-run/short-run component model in the dynamic equations to separate out transitory movements in volatility.

\section{A Matrix Algebra and Matrix Calculus}

Most of the results in this appendix can be found in Magnus (1988) and Magnus and Neudecker (1999). The vec operator stacks the columns of a $(k \times k)$ matrix into a $\left(k^{2} \times 1\right)$ vector. The vech operator stacks the lower triangular part including the main diagonal of a $(k \times k)$ symmetric matrix into a $\left(k^{*} \times 1\right)$ vector, $k^{*}=k(k+1) / 2$.

For any matrices $A(k \times l), B(l \times m)$ and $C(m \times n), \operatorname{vec}(A B C)=\left(C^{\prime} \otimes A\right) \operatorname{vec}(B)$. For any $(k \times k)$ matrix $A$, the $\left(k^{*} \times k^{2}\right)$ elimination matrix $L_{k}$ is defined such that $\operatorname{vech}(A)=L_{k} \operatorname{vec}(A)$, while for any $(k \times k)$ symmetric matrix $A$, the $\left(k^{2} \times k^{*}\right)$ duplication matrix $D_{k}$ is defined such that $\operatorname{vec}(A)=D_{k} \operatorname{vech}(A)$. Let $\Lambda_{i j}$ be a $(k \times k)$ matrix with 1 in its $(i, j)^{t h}$ position and zeros elsewhere. Then $L_{k}$ and $D_{k}$ can be obtained using $L_{k}=\sum_{i \geq j} \operatorname{vech}\left(\Lambda_{i j}\right)\left(\operatorname{vec}\left(\Lambda_{i j}\right)\right)^{\prime}$ and $D_{k}=$ $\sum_{i>j} \operatorname{vec}\left(\Lambda_{i j}+\Lambda_{j i}\right)\left(\operatorname{vech}\left(\Lambda_{i j}\right)\right)^{\prime}+\sum_{i=j} \operatorname{vec}\left(\Lambda_{i j}\right)\left(\operatorname{vech}\left(\Lambda_{i j}\right)\right)^{\prime}$.

The above results can be combined to give the following result for any $(k \times k)$ matrices $A, B$ and $C$ with $B$ being symmetric

$$
\operatorname{vech}(A B C)=L_{k} \operatorname{vec}(A B C)=L_{k}\left(C^{\prime} \otimes A\right) \operatorname{vec}(B)=L_{k}\left(C^{\prime} \otimes A\right) D_{k} \operatorname{vech}(B) .
$$

For a $(k \times l)$ matrix function $F(X)$ and a $(m \times n)$ matrix of variables $X$, the derivative of $F(X)$ with respect to $X$, denoted by the $(k l \times m n)$ matrix $D F(X)$, is given by

$$
D F(X)=\frac{\partial \operatorname{vec}(F(X))}{\partial(\operatorname{vec}(X))^{\prime}} .
$$

Also for any $(k \times k)$ matrix $A$,

$$
\frac{\partial A^{-1}}{\partial A}=-\left(\left(A^{-1}\right)^{\prime} \otimes A^{-1}\right), \frac{\partial \log |A|}{\partial A}=\left(\operatorname{vec}\left(\left(A^{-1}\right)^{\prime}\right)\right)^{\prime}, \text { and } \frac{\partial \operatorname{tr}(A X)}{\partial X}=\left(\operatorname{vec}\left(A^{\prime}\right)\right)^{\prime} .
$$

\section{B Second Moments' Structure}

Since the model is expressed for $p_{t}$ (i.e. for the squares and cross-products of daily returns), we are able to obtain explicit expressions for the fourth moment of returns by deriving $\operatorname{Var}\left[p_{t}\right]$. Similarly, 
by deriving $\operatorname{Var}\left[v_{t}\right]$, we are able to analyze the second moment of the realized measure which gives an expression for the volatility of volatility; see Engle (2002) and Corsi et al. (2008) for a discussion of modelling the volatility of volatility using the VIX and realized volatility, respectively.

The following proposition gives the structure of second moments of the matrix of outer product of daily returns and the realized measure, which is derived under the assumption that conditional moment assumptions that $\mathrm{E}_{t-1}\left[\varepsilon_{t}\right]=\mathrm{E}_{t-1}\left[\eta_{t}\right]=I_{k}$ hold. The expressions in equations (B.1) and (B.2) can be simplified further by assuming a Wishart distribution for the innovation matrices $\varepsilon_{t}$ and $\eta_{t}$, which gives (B.3) and (B.4).

Proposition 4 (i) Under the assumption that $\varepsilon_{t}$ and $\eta_{t}$ obey the conditional moment assumptions, $\mathrm{E}_{t-1}\left[\varepsilon_{t}\right]=\mathrm{E}_{t-1}\left[\eta_{t}\right]=I_{k}$, the second moments of $p_{t}$ and $v_{t}$ are given by

$$
\begin{aligned}
\operatorname{Var}\left[p_{t}\right] & =\mathrm{E}\left[Z_{H, t} \operatorname{Var}_{t-1}\left[\operatorname{vech}\left(\varepsilon_{t}\right)\right] Z_{H, t}^{\prime}\right]+\operatorname{Var}\left[Z_{H, t} \operatorname{vech}\left(I_{k}\right)\right], \\
\operatorname{Var}\left[v_{t}\right] & =\mathrm{E}\left[Z_{M, t} \operatorname{Var}_{t-1}\left[\operatorname{vech}\left(\eta_{t}\right)\right] Z_{M, t}^{\prime}\right]+\operatorname{Var}\left[Z_{M, t} \operatorname{vech}\left(I_{k}\right)\right],
\end{aligned}
$$

where $Z_{H, t}=L_{k}\left(H_{t}^{\frac{1}{2}} \otimes H_{t}^{\frac{1}{2}}\right) D_{k}$ and $Z_{M, t}=L_{k}\left(M_{t}^{\frac{1}{2}} \otimes M_{t}^{\frac{1}{2}}\right) D_{k}$.

(ii) Under the additional assumption that $\varepsilon_{t}$ and $\eta_{t}$ are i.i.d. Wishart distributed, the second moments of $p_{t}$ and $v_{t}$ are given by

$$
\begin{gathered}
\mathrm{E}\left[p_{t} p_{t}^{\prime}\right]=2 D_{k}^{+} \mathrm{E}\left[\left(H_{t} \otimes H_{t}\right)\right] L_{k}^{\prime}+\mathrm{E}\left[h_{t} h_{t}^{\prime}\right], \\
\mathrm{E}\left[v_{t} v_{t}^{\prime}\right]=2 D_{k}^{+} \mathrm{E}\left[\left(M_{t} \otimes M_{t}\right)\right] L_{k}^{\prime}+\mathrm{E}\left[m_{t} m_{t}^{\prime}\right],
\end{gathered}
$$

where $D_{k}^{+}=\left(D_{k}^{\prime} D_{k}\right)^{-1} D_{k}^{\prime}$ is the Moore-Penrose inverse of the duplication matrix, $D_{k}$.

Dropping the $t$ subscripts to avoid cluttered notation, the second moment structure of $p_{t}$ given in (B.3) will have the following structure in the 2-dimensional case

$$
\mathrm{E}\left[p p^{\prime}\right]=\mathrm{E}\left(\begin{array}{ccc}
r_{1}^{4} & r_{1}^{3} r_{2} & r_{1}^{2} r_{2}^{2} \\
r_{1}^{3} r_{2} & r_{1}^{2} r_{2}^{2} & r_{1} r_{2}^{3} \\
r_{1}^{2} r_{2}^{2} & r_{1} r_{2}^{3} & r_{2}^{4}
\end{array}\right)=\mathrm{E}\left(\begin{array}{ccc}
3 h_{11}^{2} & 3 h_{11} h_{12} & 2 h_{12}^{2}+h_{11} h_{22} \\
3 h_{11} h_{21} & 2 h_{21}^{2}+h_{11} h_{22} & 3 h_{12} h_{22} \\
2 h_{21}^{2}+h_{11} h_{22} & 3 h_{21} h_{22} & 3 h_{22}^{2}
\end{array}\right),
$$

where lower case letters, $r_{1}$ and $r_{2}$, are the daily returns for assets 1 and 2 , respectively, and $h_{i j}$, $i, j=1,2$, are the elements of $H_{t}$. Applying a vec operator to (B.3) gives a similar result to (10) in Hafner (2003), which discusses the fourth moment structure of GARCH models when $H_{t}$ follows a GARCH specification and daily returns are assumed to be normally distributed.

The result in equation (B.4) seems novel in the context of realized measures. In the univariate case, Corsi et al. (2008) estimate the volatility of realized volatility by utilizing consistent estimators of the integrated quarticity of returns, such as realized quarticity, realized quad-power quarticity and realized tri-power quarticity. In an application to S\&P 500 index futures, they show that the unconditional distributions of these three measures exhibit skewness and leptokurtosis even after applying a log transformation. In addition, they also exhibit time variation and volatility clustering, which prompts the authors to develop a GARCH-type model for realized volatility. Engle (2002) also discusses different models for the volatility of volatility using the VIX time series. 


\section{The Wishart Distribution: An Overview}

The Wishart distribution is the matrix-variate generalization of the $\chi^{2}$ distribution. It is the sampling distribution of the sample covariance matrix for random draws from the multivariate normal distribution; see Gupta and Nagar (2000) for a detailed treatment. We begin our overview by relating the Wishart distribution to the multivariate normal and the matrix-variate normal. Let $x_{1}, \ldots, x_{n}$ be independent random vectors drawn from the (centered) multivariate normal distribution where each $(k \times 1)$ vector $x_{i} \sim N_{k}(0, \Sigma), i=1, \ldots, n$. Then the $(k \times n)$ matrix $X=\left(x_{1}, \ldots, x_{n}\right)$ has a matrix variate normal distribution denoted as $X \sim N_{k, n}\left(0, \Sigma \otimes I_{\mathbf{n}}\right)$. If $n \geq k$, then $S=X X^{\prime}$ is a positive definite $(k \times k)$ matrix and follows a (centered) Wishart distribution, denoted as $S \sim$ $W_{k}(n, \Sigma)$, where $n$ is integer degrees of freedom and $\Sigma$ is the scale matrix. The unconditional moments of $S$ are given by $\mathrm{E}(S)=n \Sigma$, and $\operatorname{Var}(\operatorname{vec}(S))=2 n D_{k} D_{k}^{+}(\Sigma \otimes \Sigma)$.

The density of $S$ is given by

$$
W_{k}(n, \Sigma)=\frac{|S|^{\frac{n-k-1}{2}}}{2^{\frac{n k}{2}} \Gamma_{k}\left(\frac{n}{2}\right)|\Sigma|^{\frac{n}{2}}} \exp \left(-\frac{1}{2} \operatorname{tr}\left(\Sigma^{-1} S\right)\right), \quad n \geq k,
$$

where $\Gamma_{k}\left(\frac{n}{2}\right)=\pi^{k(k-1) / 4} \prod_{j=1}^{k} \Gamma\left(\frac{n}{2}+(1-j) / 2\right)$ is the multivariate gamma function. It is also useful to define a standardized Wishart distribution such that if $S \sim S W_{k}(n, \Sigma)$, where $S W_{k}$ denote a standardized Wishart distribution of dimension $k$, we have that $\mathrm{E}(S)=\Sigma$ instead of $\mathrm{E}(S)=n \Sigma$ under the non-standardized Wishart. Note that $S W_{k}(n, \Sigma)$ is equivalent to $W_{k}\left(n, n^{-1} \Sigma\right)$. In this case, the density of $S$ is given by

$$
S W_{k}(n, \Sigma)=\frac{n^{\frac{n k}{2}}|S|^{\frac{n-k-1}{2}}}{2^{\frac{n k}{2}} \Gamma_{k}\left(\frac{n}{2}\right)|\Sigma|^{\frac{n}{2}}} \exp \left(-\frac{n}{2} \operatorname{tr}\left(\Sigma^{-1} S\right)\right), \quad n \geq k .
$$

If the $(k \times n)$ matrix $X$ does not have full column rank (i.e. $n<k), S=X X^{\prime}$ follows instead a singular Wishart distribution; see Uhlig (1994) and Srivastava (2003). Uhlig (1994) derives the density of the singular Wishart as

$$
S I N G W_{k}(n, \Sigma)=\frac{\pi^{\left(-k n+n^{2}\right) / 2}|\widetilde{S}|^{\frac{n-k-1}{2}}}{2^{\frac{n k}{2}} \Gamma_{n}\left(\frac{n}{2}\right)|\Sigma|^{\frac{n}{2}}} \exp \left(-\frac{1}{2} \operatorname{tr}\left(\Sigma^{-1} S\right)\right), \quad n<k
$$

where $\widetilde{S}$ is a diagonal matrix containing the non-zero eigenvalues of $S$ along the main diagonal.

One of the key results on Wishart distributions is that if $S \sim W_{k}(n, \Sigma)$, then $A S A^{\prime} \sim W_{k}\left(n, A \Sigma A^{\prime}\right)$ for any $(k \times k)$ nonsingular matrix $A$. Srivastava (2003) extends this result to the singular Wishart case. Based on the ranks of $P_{t}$ and $V_{t}$, we use this result in specifying the distribution of the innovation matrices $\varepsilon_{t}$ and $\eta_{t}$ as discussed in 3.1 . 


\section{Technical Proofs}

\section{D.1 Proof of Proposition 1}

By taking unconditional expectation of (7) and (8) we have

$$
\omega_{H}=C_{H}+B_{H} \omega_{H}+A_{H} \omega_{M}, \quad \omega_{M}=C_{M}+B_{M} \omega_{M}+A_{M} \omega_{M} .
$$

By definition $\bar{\kappa}=\Omega_{M}^{\frac{1}{2}} \Omega_{H}^{-\frac{1}{2}}$, which implies $\Omega_{M}^{\frac{1}{2}}=\bar{\kappa} \Omega_{H}^{\frac{1}{2}}$, and $\Omega_{M}=\Omega_{M}^{\frac{1}{2}} \Omega_{M}^{\frac{1}{2}}=\bar{\kappa} \Omega_{H} \bar{\kappa}^{\prime}$ using the fact that $\Omega_{M}^{\frac{1}{2}}$ and $\Omega_{H}^{\frac{1}{2}}$ are symmetric since we obtain the matrix square root by the spectral decomposition. Thus $\omega_{M}:=\operatorname{vech}\left(\Omega_{M}\right)=\operatorname{vech}\left(\bar{\kappa} \Omega_{H} \bar{\kappa}^{\prime}\right)=L_{k}(\bar{\kappa} \otimes \bar{\kappa}) D_{k} \omega_{H}$ using (A.1) for the last equality.

Let $\kappa=L_{k}(\bar{\kappa} \otimes \bar{\kappa}) D_{k}$ and substitute the last result for $\omega_{M}$ in the first expression in (D.1). By collecting terms we have that the intercept coefficients are given by $C_{H}=\left(I_{k^{*}}-B_{H}-A_{H} \kappa\right) \omega_{H}$ and $C_{M}=\left(I_{k^{*}}-B_{M}-A_{M}\right) \omega_{M}$, which when substituted in (7) and (8) gives the stated result.

The proof for (11) follows by noting that $\mathrm{E}\left[\widetilde{v}_{t}\right]=\kappa^{-1} \mathrm{E}\left[v_{t}\right]=\kappa^{-1} \omega_{M}=\omega_{H}$, where the last equality follows from above by defining $\kappa=L_{k}(\bar{\kappa} \otimes \bar{\kappa}) D_{k}$. The rest follows by the same arguments in the proof of (8).

\section{D.2 Proof of Proposition 2}

We start with the proof of (14). The 1-step forecast of $h_{t}$ is $\mathrm{E}_{t}\left[h_{t+1}\right]=h_{t+1}$, since $h_{t+1}$ is $\mathcal{F}_{t}^{H F_{-}}$ measurable. From (7), the 2-step forecast is

$$
\mathrm{E}_{t}\left[h_{t+2}\right]=\mathrm{E}_{t}\left[C_{H}+B_{H} h_{t+1}+A_{H} v_{t+1}\right]=C_{H}+B_{H} h_{t+1}+A_{H} \mathrm{E}_{t}\left[v_{t+1}\right] .
$$

The 3-step forecast is

$$
\begin{aligned}
\mathrm{E}_{t}\left[h_{t+3}\right] & =\mathrm{E}_{t}\left[C_{H}+B_{H} h_{t+2}+A_{H} v_{t+2}\right]=C_{H}+B_{H} \mathrm{E}_{t}\left[h_{t+2}\right]+A_{H} \mathrm{E}_{t}\left[v_{t+2}\right] \\
& =\left(I_{k^{*}}+B_{H}\right) C_{H}+B_{H}^{2} h_{t+1}+A_{H} \mathrm{E}_{t}\left[v_{t+2}\right]+B_{H} A_{H} \mathrm{E}_{t}\left[v_{t+1}\right],
\end{aligned}
$$

where the last equality follows by substituting for $\mathrm{E}_{t}\left[h_{t+2}\right]$ from above and collecting terms. By forward iteration, it is straightforward to show that

$$
\mathrm{E}_{t}\left[h_{t+s}\right]=\sum_{i=1}^{s-1} B_{H}^{i-1} C_{H}+B_{H}^{s-1} h_{t+1}+\sum_{i=1}^{s-1} B_{H}^{i-1} A_{H} \mathrm{E}_{t}\left[v_{t+s-i}\right] .
$$

Now find an expression for $\mathrm{E}_{t}\left[v_{t+s-i}\right]$ in terms of $m_{t+1}$, which is $\mathcal{F}_{t}^{H F}$-measurable. We start with the 1-step forecast of $v_{t}$ which is $\mathrm{E}_{t}\left[v_{t+1}\right]=m_{t+1}$ by definition. The 2-step forecast is

$$
\begin{aligned}
\mathrm{E}_{t}\left[v_{t+2}\right] & =\mathrm{E}_{t}\left[\mathrm{E}_{t+1}\left[v_{t+2}\right]\right]=\mathrm{E}_{t}\left[m_{t+2}\right]=\mathrm{E}_{t}\left[C_{M}+B_{M} m_{t+1}+A_{M} v_{t+1}\right] \\
& =C_{M}+\left(B_{M}+A_{M}\right) m_{t+1},
\end{aligned}
$$


since $\mathrm{E}_{t}\left[v_{t+1}\right]=m_{t+1}$. The 3 -step forecast is

$$
\begin{aligned}
\mathrm{E}_{t}\left[v_{t+3}\right] & =\mathrm{E}_{t}\left[\mathrm{E}_{t+1}\left[v_{t+3}\right]\right]=\mathrm{E}_{t}\left[C_{M}+\left(B_{M}+A_{M}\right) m_{t+2}\right]=C_{M}+\left(B_{M}+A_{M}\right) \mathrm{E}_{t}\left[m_{t+2}\right] \\
& =\left(I_{k^{*}}+\left(B_{M}+A_{M}\right)\right) C_{M}+\left(B_{M}+A_{M}\right)^{2} m_{t+1},
\end{aligned}
$$

where the second equality follows by substitution from above with a 1-period forward iteration, and the last equality follows by substituting for $m_{t+2}$, applying the expectations operator and collecting terms. By forward iteration, we have the following formula for the $s$-step forecast of $v_{t}$

$$
\mathrm{E}_{t}\left[v_{t+s}\right]=\sum_{j=1}^{s-1}\left(B_{M}+A_{M}\right)^{j-1} C_{M}+\left(B_{M}+A_{M}\right)^{s-1} m_{t+1} .
$$

Using (D.3) to substitute for $\mathrm{E}_{t}\left[v_{t+s-i}\right]$ in (D.2), while adapting the summation limits by replacing $s$ in (D.3) with $(s-i)$ gives the stated result.

The proof of (15) follows similar steps. We start by taking unconditional expectations of (7) and (8) which gives

$$
\omega_{H}=C_{H}+B_{H} \omega_{H}+A_{H} \omega_{M}, \quad \omega_{M}=C_{M}+B_{M} \omega_{M}+A_{M} \omega_{M},
$$

so that the constant terms can be expressed as $C_{H}=\omega_{H}-B_{H} \omega_{H}-A_{H} \omega_{M}$ and $C_{M}=\omega_{M}-$ $B_{M} \omega_{M}-A_{M} \omega_{M}$. Substituting these expressions in (7) and (8) gives

$$
\begin{aligned}
h_{t} & =\omega_{H}-B_{H} \omega_{H}-A_{H} \omega_{M}+B_{H} h_{t-1}+A_{H} v_{t-1} \\
& =\omega_{H}+B_{H}\left(h_{t-1}-\omega_{H}\right)+A_{H}\left(v_{t-1}-\omega_{M}\right), \\
m_{t} & =\omega_{M}-B_{M} \omega_{M}-A_{M} \omega_{M}+B_{M} m_{t-1}+A_{M} v_{t-1} \\
& =\omega_{M}+B_{M}\left(m_{t-1}-\omega_{M}\right)+A_{M}\left(v_{t-1}-\omega_{M}\right) .
\end{aligned}
$$

Forward iteration of these equations as illustrated in the proof of (14) yields (15).

\section{D.3 Proof of Proposition 3}

We derive the score vector and prove that it is a martingale difference sequence only for the HEAVY$\mathrm{P}$ equation. The derivation for the HEAVY-V equation is analogous. We derive the $\left(1 \times \delta_{H}\right)$ score vector $\frac{\partial l_{H, t}\left(\theta_{H}\right)}{\partial \theta_{H}}$ from the log-likelihood equation which gives

$$
\begin{aligned}
\frac{\partial l_{H, t}\left(\theta_{H}\right)}{\partial \theta_{H}^{\prime}} & =-\frac{1}{2} \frac{\partial \log \left|H_{t}\right|}{\partial \theta_{H}^{\prime}}-\frac{1}{2} \frac{\partial \operatorname{tr}\left(H_{t}^{-1} P_{t}\right)}{\partial \theta_{H}^{\prime}} \\
& =-\frac{1}{2} \frac{\partial \log \left|H_{t}\right|}{\partial H_{t}} \frac{\partial H_{t}}{\partial \theta_{H}^{\prime}}-\frac{1}{2} \frac{\partial \operatorname{tr}\left(H_{t}^{-1} P_{t}\right)}{\partial H_{t}^{-1}} \frac{\partial H_{t}^{-1}}{\partial H_{t}} \frac{\partial H_{t}}{\partial \theta_{H}^{\prime}} \\
& =-\frac{1}{2}\left(\operatorname{vec}\left(H_{t}^{-1}\right)\right)^{\prime} \frac{\partial \operatorname{vec}\left(H_{t}\right)}{\partial \theta_{H}^{\prime}}+\frac{1}{2}\left(\operatorname{vec}\left(P_{t}\right)\right)^{\prime}\left(H_{t}^{-1} \otimes H_{t}^{-1}\right) \frac{\partial \operatorname{vec}\left(H_{t}\right)}{\partial \theta_{H}^{\prime}}
\end{aligned}
$$




$$
\begin{aligned}
& =\frac{1}{2}\left[\left(\operatorname{vec}\left(P_{t}\right)\right)^{\prime}\left(H_{t}^{-1} \otimes H_{t}^{-1}\right)-\left(\operatorname{vec}\left(H_{t}^{-1}\right)\right)^{\prime}\right] \frac{\partial \operatorname{vec}\left(H_{t}\right)}{\partial \theta_{H}^{\prime}} \\
& =\frac{1}{2}\left[\left(\operatorname{vec}\left(P_{t}\right)\right)^{\prime}\left(H_{t}^{-1} \otimes H_{t}^{-1}\right)-\left(\operatorname{vec}\left(H_{t}^{-1} H_{t} H_{t}^{-1}\right)\right)^{\prime}\right] \frac{\partial v e c\left(H_{t}\right)}{\partial \theta_{H}^{\prime}} \\
& =\frac{1}{2}\left[\left(\operatorname{vec}\left(P_{t}\right)\right)^{\prime}\left(H_{t}^{-1} \otimes H_{t}^{-1}\right)-\left(\left(H_{t}^{-1} \otimes H_{t}^{-1}\right) \operatorname{vec}\left(H_{t}\right)\right)^{\prime}\right] \frac{\partial \operatorname{vec}\left(H_{t}\right)}{\partial \theta_{H}^{\prime}} \\
& =\frac{1}{2}\left[\left(\operatorname{vec}\left(P_{t}\right)\right)^{\prime}-\left(\operatorname{vec}\left(H_{t}\right)\right)^{\prime}\right]\left(H_{t}^{-1} \otimes H_{t}^{-1}\right) \frac{\partial \operatorname{vec}\left(H_{t}\right)}{\partial \theta_{H}^{\prime}},
\end{aligned}
$$

where in the second equality we used the chain rule and the matrix derivatives in the third equality are obtained using the rules stated in Appendix A.

The score vector is a martingale difference sequence such that $\mathrm{E}_{t-1}\left[\frac{\partial l_{H, t}\left(\theta_{H}\right)}{\partial \theta_{H}^{\prime}}\right]=0$ as the conditional expectation of the term in square brackets is 0 since

$$
\left.\mathrm{E}_{t-1}\left[\left(\operatorname{vec}\left(P_{t}\right)\right)^{\prime}-\left(\operatorname{vec}\left(H_{t}\right)\right)^{\prime}\right]=\mathrm{E}_{t-1}\left[\left(\operatorname{vec}\left(P_{t}\right)\right)^{\prime}\right]-\mathrm{E}_{t-1}\left(\operatorname{vec}\left(H_{t}\right)\right)^{\prime}\right]=0,
$$

where we use $\mathrm{E}_{t-1}\left[\left(\operatorname{vec}\left(P_{t}\right)\right)^{\prime}\right]=\left(\operatorname{vec}\left(H_{t}\right)\right)^{\prime}$, which follows directly by applying a vec operator to (3) and using the conditional moment assumption $\mathrm{E}_{t-1}\left[\varepsilon_{t}\right]=I_{k}$.

\section{D.4 Proof of Proposition 4}

We only show the proof for (B.1) and (B.3), as (B.2) and (B.4) follows similar arguments. We start from $p_{t}:=\operatorname{vech}\left(P_{t}\right)=\operatorname{vech}\left(H_{t}^{\frac{1}{2}} \varepsilon_{t} H_{t}^{\frac{1}{2}}\right)=L_{k}\left(H_{t}^{\frac{1}{2}} \otimes H_{t}^{\frac{1}{2}}\right) D_{k} v e c h\left(\varepsilon_{t}\right)$, where the last result follows from (A.1). Let $Z_{H, t}=L_{k}\left(H_{t}^{\frac{1}{2}} \otimes H_{t}^{\frac{1}{2}}\right) D_{k}$, which is $\mathcal{F}_{t-1}^{H F}$-measurable. Also, let $\operatorname{Var}_{t}[\cdot]:=\operatorname{Var}\left[\cdot \mid \mathcal{F}_{t}^{H F}\right]$ denote the variance conditional on $\mathcal{F}_{t}^{H F}$. Using the variance decomposition, we obtain

$$
\begin{aligned}
V\left[p_{t}\right] & =\mathrm{E}\left[\operatorname{Var}_{t-1}\left[p_{t}\right]\right]+\operatorname{Var}\left[\mathrm{E}_{t-1}\left[p_{t}\right]\right] \\
& =\mathrm{E}\left[\operatorname{Var}_{t-1}\left[Z_{H, t} \operatorname{vech}\left(\varepsilon_{t}\right)\right]\right]+\operatorname{Var}\left[\mathrm{E}_{t-1}\left[Z_{H, t} \operatorname{vech}\left(\varepsilon_{t}\right)\right]\right] \\
& =\mathrm{E}\left[Z_{H, t} \operatorname{Var}_{t-1}\left[\operatorname{vech}\left(\varepsilon_{t}\right)\right] Z_{H, t}^{\prime}\right]+\operatorname{Var}\left[Z_{H, t} \mathrm{E}_{t-1}\left[\operatorname{vech}\left(\varepsilon_{t}\right)\right]\right]
\end{aligned}
$$

as $Z_{H, t}$ is $\mathcal{F}_{t-1}^{H F}$-measurable. As $\mathrm{E}_{t-1}\left[\varepsilon_{t}\right]=I_{k}$ by assumption, it follows that $\mathrm{E}_{t-1}\left[\right.$ vech $\left.\left(\varepsilon_{t}\right)\right]=$ $\operatorname{vech}\left(I_{k}\right)$. Thus the last equation can be simplified to give (B.1).

For (B.3), $\varepsilon_{t} \stackrel{i . i . d .}{\sim} W_{k}\left(1, I_{k}\right)$ so $P_{t} \mid \mathcal{F}_{t-1}^{H F} \sim W_{k}\left(1, H_{t}\right)$ using the property of quadratic forms for Wishart and singular Wishart matrices; see respectively Gupta and Nagar (2000, Theorem 3.3.1) and Srivastava (2003, Corollary 3.5). Since $P_{t} \mid \mathcal{F}_{t-1}^{H F} \sim W_{k}\left(1, H_{t}\right)$, we have that $\operatorname{Var}_{t-1}\left[\operatorname{vec}\left(P_{t}\right)\right]=$ $2 D_{k} D_{k}^{+}\left(H_{t} \otimes H_{t}\right)$ where $D_{k}$ is the duplication matrix, and $D_{k}^{+}=\left(D_{k}^{\prime} D_{k}\right)^{-1} D_{k}^{\prime}$ is its Moore-Penrose inverse. This result follows from Magnus (1988, Theorem 10.1) by noting that conditioning on $\mathcal{F}_{t-1}^{H F}$ enables us to treat $H_{t}$ as a nonstochastic matrix. Therefore

$$
\begin{aligned}
\operatorname{Var}_{t-1}\left[p_{t}\right] & =\operatorname{Var}_{t-1}\left[L_{k} \operatorname{vec}\left(P_{t}\right)\right]=L_{k} \operatorname{Var}_{t-1}\left[\operatorname{vec}\left(P_{t}\right)\right] L_{k}^{\prime} \\
& =2 L_{k} D_{k} D_{k}^{+}\left(H_{t} \otimes H_{t}\right) L_{k}^{\prime}=2 D_{k}^{+}\left(H_{t} \otimes H_{t}\right) L_{k}^{\prime}
\end{aligned}
$$


where the last equality follows since $L_{k} D_{k}=I_{k^{*}}$ by Magnus (1988, Theorem 5.5). We obtain the unconditional second moment of $p_{t}$ using the variance decomposition

$$
\begin{aligned}
\operatorname{Var}\left[p_{t}\right] & =\mathrm{E}\left[\operatorname{Var}_{t-1}\left[p_{t}\right]\right]+\operatorname{Var}\left[\mathrm{E}_{t-1}\left[p_{t}\right]\right]=\mathrm{E}\left[2 D_{k}^{+}\left(H_{t} \otimes H_{t}\right) L_{k}^{\prime}\right]+\operatorname{Var}\left[h_{t}\right] \\
& =2 D_{k}^{+} \mathrm{E}\left[\left(H_{t} \otimes H_{t}\right)\right] L_{k}^{\prime}+\operatorname{Var}\left[h_{t}\right] .
\end{aligned}
$$

We can write $\operatorname{Var}\left[p_{t}\right]=\mathrm{E}\left[p_{t} p_{t}^{\prime}\right]-\mathrm{E}\left[p_{t}\right] \mathrm{E}\left[p_{t}\right]^{\prime}$ and $\operatorname{Var}\left[h_{t}\right]=\mathrm{E}\left[h_{t} h_{t}^{\prime}\right]-\mathrm{E}\left[h_{t}\right] \mathrm{E}\left[h_{t}\right]^{\prime}$. By noting that $\mathrm{E}\left[p_{t}\right]=\mathrm{E}\left[\mathrm{E}_{t-1}\left[p_{t}\right]\right]=\mathrm{E}\left[h_{t}\right]$, we can simplify the last equation to

$$
\mathrm{E}\left[p_{t} p_{t}^{\prime}\right]=2 D_{k}^{+} \mathrm{E}\left[\left(H_{t} \otimes H_{t}\right)\right] L_{k}^{\prime}+\mathrm{E}\left[h_{t} h_{t}^{\prime}\right]
$$

which gives the stated result.

\section{References}

Amisano, G. and R. Giacomini (2007). Comparing density forecasts via weighted likelihood ratio tests. Journal of Business 83 Economic Statistics 25, 177-190.

Andersen, T. G. and T. Bollerslev (1998). Answering the skeptics: yes, standard volatility models do provide accurate forecasts. International Economic Review 39, 885-905.

Andersen, T. G., T. Bollerslev, and F. X. Diebold (2007). Roughing it up: Including jump components in the measurement, modeling and forecasting of return volatility. Review of Economics and Statistics 89, $707-720$

Andersen, T. G., T. Bollerslev, F. X. Diebold, and P. Labys (2003). Modeling and forecasting realized volatility. Econometrica 71, 579-625.

Andersen, T. G., T. Bollerslev, and N. Meddahi (2011). Market microstructure noise and realized volatility forecasting. Journal of Econometrics 160, 220-234.

Asai, M., M. McAleer, and J. Yu (2006). Multivariate stochastic volatility: a review. Econometric Reviews 25, $145-175$.

Barndorff-Nielsen, O. E., P. R. Hansen, A. Lunde, and N. Shephard (2008). Designing realised kernels to measure the ex-post variation of equity prices in the presence of noise. Econometrica 76, 1481-1536.

Barndorff-Nielsen, O. E., P. R. Hansen, A. Lunde, and N. Shephard (2011). Multivariate realised kernels: consistent positive semi-definite estimators of the covariation of equity prices with noise and non-synchronous trading. Journal of Econometrics. Forthcoming.

Barndorff-Nielsen, O. E. and N. Shephard (2002). Econometric analysis of realised volatility and its use in estimating stochastic volatility models. Journal of the Royal Statistical Society, Series B 64, 253-280.

Barndorff-Nielsen, O. E. and N. Shephard (2004). Econometric analysis of realised covariation: high frequency covariance, regression and correlation in financial economics. Econometrica 72, 885-925. 
Bauer, G. H. and K. Vorkink (2011). Forecasting multivariate realized stock market volatility. Journal of Econometrics 160, 93-101.

Bauwens, L., S. Laurent, and J. V. K. Rombouts (2006). Multivariate GARCH models: A survey. Journal of Applied Econometrics 21, 79-109.

Bollerslev, T. and J. M. Wooldridge (1992). Quasi maximum likelihood estimation and inference in dynamic models with time varying covariances. Econometric Reviews 11, 143-172.

Brownlees, C. T. and R. F. Engle (2010). Volatility, correlation and tails for systemic risk measurment. Working paper: Stern School of Business, New York University.

Brownlees, C. T. and G. M. Gallo (2010). Comparison of volatility measures: a risk management perspective. Journal of Financial Econometrics 8, 29-56.

Chib, S., Y. Omori, and M. Asai (2009). Multivariate stochastic volatility. In T. G. Andersen, R. A. Davis, J. P. Kreiss, and T. Mikosch (Eds.), Handbook of Financial Time Series, pp. 365-400. Springer-Verlag.

Chiriac, R. and V. Voev (2011). Modelling and forecasting multivariate realized volatility. Journal of Applied Econometrics. forthcoming.

Cipollini, F., R. F. Engle, and G. Gallo (2007). A model for multivariate non-negative valued processes in financial econometrics. Unpublished paper: Stern School of Business, New York University.

Comte, F. and O. Lieberman (2003). Asymptotic theory for multivariate GARCH processes. Journal of Multivariate Analysis 84, 61-84.

Corsi, F. S., S. Mittnik, C. Pigorsch, and U. Pigorsch (2008). The volatility of realized volatility. Econometric Reviews 17, 46-78.

Cox, D. R. (1961). Tests of separate families of hypotheses. Proceedings of the Berkeley Symposium 4, $105-123$.

Diebold, F. X. and R. S. Mariano (1995). Comparing predictive accuracy. Journal of Business $\mathscr{E}$ Economic Statistics 13, 253-263.

Diks, C., V. Panchenko, and D. van Dijk (2008). Out-of-sample comparison of copula specifications in multivariate density forecasts. Working paper: Australian School of Business, University of New South Wales.

Engle, R. F. (2002). New frontiers for ARCH models. Journal of Applied Econometrics 17, 425-446.

Engle, R. F. and J. P. Gallo (2006). A multiple indicator model for volatility using intra daily data. Journal of Econometrics 131, 3-27.

Engle, R. F., D. F. Hendry, and J. F. Richard (1983). Exogeneity. Econometrica 51, 277-304.

Engle, R. F. and K. F. Kroner (1995). Multivariate simultaneous generalized ARCH. Econometric Theory 11, 122-150. 
Engle, R. F. and J. Mezrich (1996). GARCH for groups. Risk 9, 36-40.

Giacomini, R. and H. White (2006). Tests of conditional predictive ability. Econometrica 74, 1545-1578.

Gupta, A. K. and D. K. Nagar (2000). Matrix Variate Distributions. New York: Chapman \& Hall.

Hafner, C. M. (2003). Fourth moment structure of multivariate GARCH models. Journal of Financial Econometrics 1, 26-54.

Hafner, C. M. and A. Preminger (2009). On asymptotic theory for multivariate GARCH models. Journal of Multivariate Analysis 100, 2044-2054.

Hansen, P. R., Z. A. Huang, and H. H. Shek (2011). Realized GARCH: a complete model of returns and realized measures of volatility. Journal of Applied Econometrics. forthcoming.

Hansen, P. R., A. Lunde, and V. Voev (2010). Realized beta GARCH: a multivariate GARCH model with realized measures of volatility and covolatility. Working paper: CREATES, Aarhus University.

Jeantheau, T. (1998). Strong consistency of estimators for multivariate ARCH models. Econometric Theory $14,70-86$.

Jin, X. and J. M. Maheu (2010). Modelling realized covariances and returns. Working paper: Department of Economics, University of Toronto.

Laurent, S., J. V. K. Rombouts, and F. Violante (2009). On loss functions and ranking forecasting performances of multivariate volatility models. Working paper.

Lutkepohl, H. (1996). Handbook of Matrices. Chichester: Wiley.

Magnus, J. R. (1988). Linear Structures. London: Griffin.

Magnus, J. R. and H. Neudecker (1999). Matrix Differential Calculus with Applications in Statistics and Econometrics. New York: Wiley. revised edition.

Nelsen, R. B. (2006). An Introduction to Copulas (2 ed.). New York: Springer.

Newey, W. K. and D. McFadden (1994). Large sample estimation and hypothesis testing. In R. F. Engle and D. McFadden (Eds.), The Handbook of Econometrics, Volume 4, pp. 2111-2245. North-Holland.

Newey, W. K. and K. D. West (1987). A simple positive semi-definite, heteroskedasticity and autocorrelation consistent covariance matrix. Econometrica 55, 703-708.

Patton, A. J. (2009). Copula-based models for financial time series. In T. G. Andersen, R. A. Davis, J. P. Kreiss, and T. Mikosch (Eds.), Handbook of Financial Time Series, pp. 767-785. Springer-Verlag.

Patton, A. J. (2011). Volatility forecast comparison using imperfect volatility proxies. Journal of Econometrics $160,246-256$.

Patton, A. J. and K. K. Sheppard (2009). Evaluating volatility and correlation forecasts. In T. G. Andersen, R. A. Davis, J. P. Kreiss, and T. Mikosch (Eds.), Handbook of Financial Time Series, pp. 801-838. Springer Verlag. 
Shephard, N. and K. K. Sheppard (2010). Realising the future: forecasting with high-frequency-based volatility (HEAVY) models. Journal of Applied Econometrics 25, 197-231.

Srivastava, M. S. (2003). Singular Wishart and multivariate Beta distributions. Annals of Statistics 31, $1537-1560$.

Uhlig, H. (1994). On singular Wishart and singular multivariate Beta distributions. Annals of Statistics 22, $395-405$.

Voev, V. (2008). Dynamic modelling of large dimensional covariance matrices. In L. Bauwens, W. Pohlmeier, and D. Veredas (Eds.), High Frequency Financial Econometrics, pp. 293-312. Physica-Verlag HD.

Vuong, Q. H. (1989). Likelihood ratio tests for model selection and non-nested hypotheses. Econometrica 57, $307-333$.

West, K. (1996). Asymptotic inference about predictive ability. Econometrica 64, 1067-1084. 